\title{
A systematic review and meta-analysis of moderate-to-vigorous physical activity levels in secondary school physical education lessons
}

Jenna L. Hollis ${ }^{1,2^{*}}$, Rachel Sutherland ${ }^{1,2,3}$, Amanda J. Williams ${ }^{1,2,3}$, Elizabeth Campbell ${ }^{1,2,3}$, Nicole Nathan ${ }^{1,2,3}$, Luke Wolfenden ${ }^{1,2,3}$, Philip J. Morgan ${ }^{4}$, David R. Lubans ${ }^{4}$, Karen Gillham ${ }^{1,2}$ and John Wiggers ${ }^{1,2,3}$

\begin{abstract}
Background: Schools play an important role in physical activity promotion for adolescents. The systematic review aimed to determine the proportion of secondary (middle and high) school physical education (PE) lesson time that students spend in moderate to vigorous physical activity (MVPA), and to assess if MVPA was moderated by school level (middle and high school), type of physical activity measurement and type of PE activities.

Methods: A systematic search of nine electronic databases was conducted (PROSPERO2014:CRD42014009649). Studies were eligible if they were published between 2005 and 2014; written in English; assessed MVPA in PE lessons of secondary (middle and high) school students; and used a quantitative MVPA measure (i.e., accelerometry, heart rate monitoring, pedometers or observational measures). Two reviewers examined the retrieved articles, assessed risk of bias, and performed data extraction. Random effects meta-analysis was used to calculate a pooled estimate of the percent of PE lesson time spent in MVPA and to assess moderator effects where data allowed.

Results: The search yielded 5,132 potentially relevant articles; 28 articles representing 25 studies (7 middle and 18 high school) from seven countries were included. Twelve studies measured MVPA through observational measures, seven used accelerometers, five used heart rate monitors and four used pedometers (including three studies using a mix of measures). Meta-analysis of 15 studies found that overall, students spent a mean (95\% Cl) of $40.5 \%$ (34.8-46.2\%) of PE in MVPA. Middle school students spent 48.6\% (41.3-55.9\%) of the lesson in MVPA ( $n=5$ studies) and high school students $35.9 \%$ (28.3-43.6\%) ( $n=10$ studies). Studies measuring MVPA using accelerometers $(n=5)$ showed that students spent 34.7\% (25.1-44.4\%) of the lesson in MVPA, while 44.4\% (38.3-50.5\%) was found for lessons assessed via observation $(n=9)$, 43.1\% (24.3-61.9\%) of the lesson for a heart rate based study, and 35.9\% (31.0-40.8\%) for a pedometermeasured study.

Conclusions: The proportion of PE spent in MVPA (40.5\%) is below the US Centre for Disease Control and Prevention and the UK Associations for Physical Education recommendation of 50\%. Findings differed according to the method of MVPA assessment. Additional strategies and intervention research are needed to build more active lesson time in PE.
\end{abstract}

Keywords: High school, Middle school, Lesson, Class, PE, Exercise, MVPA, Student, Adolescent

\footnotetext{
* Correspondence: jenna.hollis@hnehealth.nsw.gov.au

${ }^{1}$ Hunter New England Population Health, Locked Bag 10, Wallsend, NSW

2287, Australia

${ }^{2}$ School of Medicine and Public Health, University of Newcastle, Callaghan

2308, Australia

Full list of author information is available at the end of the article
}

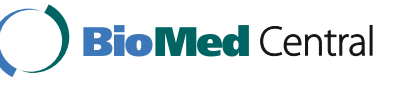

(c) The Author(s). 2017 Open Access This article is distributed under the terms of the Creative Commons Attribution 4.0 International License (http://creativecommons.org/licenses/by/4.0/), which permits unrestricted use, distribution, and reproduction in any medium, provided you give appropriate credit to the original author(s) and the source, provide a link to the Creative Commons license, and indicate if changes were made. The Creative Commons Public Domain Dedication waiver (http://creativecommons.org/publicdomain/zero/1.0/) applies to the data made available in this article, unless otherwise stated. 


\section{Background}

Moderate-to-vigorous physical activity (MVPA) during adolescence has been positively associated with a host of physiological and psychological outcomes such as cardiorespiratory fitness [1], reduced metabolic disease risk [2] and better mental health [3, 4]. The World Health Organisation (WHO) recommends children and adolescents aged 5 to 17 years old participate in $60 \mathrm{~min}$ of MVPA everyday [5]. Internationally, as few as $20 \%$ of adolescents meet this recommendation [6]. Schools play an important role in physical activity promotion for adolescents [7], an age that has been associated with declining physical activity levels $[8,9]$.

The United States' (US) Centre for Disease Control (CDC) and Prevention [10] and the United Kingdoms (UK) Associations for Physical Education (AfPE) [11] advise that children (5-17 years old) should participate in MVPA for $50 \%$ of PE lesson time to gain appropriate health and academic benefits. The most recent review to examine the proportion of PE lesson time spent in MVPA in secondary schools (i.e., middle and high school) was published in 2005 and reviewed 40 studies [12]. The majority of studies used heart rate monitoring to measure MVPA $(n=30), 10$ studies used systematic observation such as the System for Observing Fitness Instruction Time (SOFIT), and four used accelerometry [12]. When data from the studies were combined (not a meta-analysis), the review found that secondary school students engaged in MVPA for between $27 \%$ and $47 \%$ of PE time depending on the type of measurement instrument used to measure MVPA; accelerometers-assessed MVPA was reported for $46.8 \pm 13.9 \%$ of lesson time, heart rate monitor-assessed MVPA for $37.9 \pm 14.6 \%$ and observational-assessed MVPA for $26.6 \pm 15.2 \%$ of lesson time [12]. Sub-analyses found MVPA levels varied according to the type of activity students engaged in; from almost $50 \%$ of lesson time when engaged in fitness orientated activities (48\%) or team invasion games (e.g., basketball and soccer; 46\%) compared to just one third of time when participating in dance and gymnastics, or net game activities [12]. The review did not examine MVPA in middle and high school PE lessons separately.

Since 2005, numerous observational and intervention studies examining MVPA in middle and high school PE lessons have been conducted. However there has been no systematic review to examine the proportion of secondary school PE lesson time, without intervention, spent in MVPA. While it is important to acknowledge that the target of 50\% MVPA is only one aspect of assessing the quality of PE lessons, continued monitoring of guideline implementation is important as a tool to track any improvements in MVPA or achievements of PE lesson targets. Therefore, the primary aim of this systematic review was to update the evidence base and determine the proportion of secondary (middle and high) school PE lesson time that students spend in MVPA. Secondary aims were to evaluate student participation in MVPA during PE lesson time according to three potential moderators, namely: i) school level (middle or high schools); ii) type of physical activity measurement (accelerometer, heart rate monitoring, pedometry or observational measure); and iii) type of PE activities (fitness orientated activities, team invasion games, dance and gymnastics or net game activities).

\section{Methods}

\section{Search Strategy}

The systematic review protocol was registered with Prospero on the $7^{\text {th }}$ May 2014 (http://www.crd.york.ac.uk/ PROSPERO/display_record.asp?ID=CRD42014009649; PR OSPERO2014:CRD42014009649). The systematic review adhered to the Preferred Reporting Items for Systematic Reviews and Meta-analysis (PRISMA) statement [13]. A two-step search strategy was used. Firstly, a search using key words was carried out across nine electronic databases: Medline, Sport Discus, CINAHL, The Central Cochrane database of Systematic Reviews, CENTRAL, ERIC Proquest, EMBASE, Scopus and PsycINFO. Key search terms and their synonyms were searched separately in four main filters which were then combined. Search filter one identified the setting such as 'physical education,' 'lesson", 'class"'. Search filter two referred to the target population including 'child,' 'adolesc"' and 'student'. Measurement terms were identified using search filter three such as 'motor activity', 'exercise' and 'MVPA'. Search filter four identified the study design including 'prospective studies,' 'longitudinal studies', 'non-randomized'. Search terms within each filter were combined using the Boolean operator 'or', and all four filters were combined to form one search using the Boolean operator 'and'. See Additional file 1 for a record of the search strategy used for each database. In the second step of the search strategy, the reference list of the included studies was manually searched for additional papers not previously identified.

The title, abstract and description/MESH heading of the studies identified during the search were retrieved and examined by two independent reviewers (JLH and RS) to determine if the study met the inclusion criteria. The full texts of the potentially eligible studies were retrieved and independently assessed by the two reviewers for eligibility. If the two independent reviewers disagreed on whether a study should be included in the review, a third independent reviewer (EC) was consulted until a consensus was reached.

\section{Inclusion and exclusion criteria}

This review considered studies i) published in English from 2005 to 2014; ii) that assessed the physical activity 
levels of students during PE lessons at a secondary school [middle (i.e., Grade 6-8; approximately 10-14 years old) or high school (i.e., Grade 7-12; approximately 12-18 years old)]; iii) included a quantitative measure of physical activity levels (e.g., accelerometry, heart rate monitoring, pedometers or systematic observational measures); iv) were of cross sectional or prospective longitudinal quantitative design, or the baseline intervention and/or control group results of randomised controlled trials (RCTs), non-randomised controlled trials (non-RCTs) and pre-post studies. The control group results during the study period were included if no baseline control data was provided and the control group received no intervention. The review excluded studies that reported on preschool or elementary/primary school children, abstracts, theses/dissertations and unpublished literature, were published prior to 2005, and reported on only the follow-up study results from interventions in RCTs, non-RCTs and pre-post studies.

\section{Assessment of risk of bias}

An 11-item tool was developed to assess the risk of bias of the included studies (Additional file 2). The tool was created as no existing risk of bias tool assessed bias that was relevant to the topic. For example, existing tools assessed studies on participant recall bias, interviewer bias, the randomisation procedure and attrition [14-16]; all criteria which were not directly relevant to this systematic review. The existing tools lacked detailed criterion regarding selection and instrument bias across the school, class and student level, which were more likely to influence the findings. The tool consisted of seven domains covering selection bias at the school, class and student level, plus selection and instrument bias related to the PE lessons and MVPA measures. The tool was used by the authors in a previous systematic review of MVPA in elementary school PE lessons [17]. In this review, two independent reviewers (JLH and RS) used the tool to assess the risk of bias of all studies included in the systematic review. Any disagreements were resolved through discussion between the two reviewers, and if a consensus could not be reached, a third reviewer was consulted (EC). Each of the 11 criteria was coded as 'clearly described and present' (yes), 'absent' (no), or 'unclear and/or inadequately described' (unclear) for each study. Each domain was considered independently as recommended by PRISMA [13].

\section{Data collection}

Data were extracted from the retrieved papers for evidence synthesis using a pre-piloted standardised data extraction table developed by the authors. One independent reviewer (JLH) extracted the data, and a second independent review (RS) examined the completed data extraction table, added any missing information, corrected errors and highlighted any data that were unclear. The two reviewers discussed all discrepancies. A third reviewer (EC) was consulted if a consensus could not be reached. Missing data were requested from study authors if necessary to determine study eligibility and where insufficient data were provided for inclusion in the meta-analysis. The extracted data included study design, the setting (region/country, middle school, high school, school level), participants (school and student sample size, student age, sex, socioeconomic status (SES), ethnicity), teacher training, aim, recruitment, response rate, measurement type, lesson delivery, number of lessons, lesson duration, and activities engaged in during the lesson. MVPA in PE lessons was extracted as either: i) mean percentage of lesson time spent in MVPA, or ii) minutes of MVPA per lesson and length of the PE lesson so that percentage MVPA per lesson could be manually calculated. The activities engaged in during the lesson/s were extracted verbatim from the study description, with the intention to re-categorise these into four categories as in the previous review [12]: i) fitness orientated activities, ii) team invasion games, iii) dance and gymnastics, and iv) net game activities. If lessons contained activities that fell into more than one category, data on the time spent in each activity from the different categories were also extracted (if reported).

\section{Data synthesis}

Data were synthesised via a narrative description of findings from the included studies. Summaries of the physical activity levels in each study were presented as both mean $(\mathrm{SD} / \mathrm{SE} / 95 \% \mathrm{CI})$ percentage of time and actual minutes, if provided. Comprehensive Meta-analysis Software (version 2.2.064, July 2011) was used to pool the findings into a meta-analysis for studies that reported i) mean percentage of time in MVPA, ii) a standard deviation, and iii) the number of PE lessons observed. Findings were combined for the main meta-analysis regardless of the assessment method. Percentage time spent in MVPA was quantified from pedometer steps per minute by the authors (JLH and RS) using a standardised equation [18]. The meta-analysis was weighted by inverse variance assuming a random-effects model, according to the number of $\mathrm{PE}$ lessons monitored in each study. A decision to assign study weighting based on PE lessons was made as the factor variable of interest is the variability of MVPA at the lesson level, not the student level. As studies reported the measure at an aggregate lesson level (e.g., average of $39 \%$ of the lessons spent in MVPA) and the student sample size for each individual lesson was not clear for all studies, we are unable to assign study weighting by student sample size. A larger 
number of PE lessons monitored in a study would provide a more accurate estimate of percentage MVPA in usual PE lessons. Providing that either i) all students in a PE lesson are monitored, or ii) students are randomly selected for monitoring (i.e., the protocol for observational assessment using SOFIT), then the average student percentage MVPA data collected should be representative of percentage MVPA in the assessed lesson regardless of the number of students monitored.

Moderator analyses were performed to determine percent MVPA by school level (middle or high school), type of physical activity measurement (accelerometer, heart rate monitor, pedometer or observational measure) and type of PE activities (fitness orientated activities, team invasion games, dance and gymnastics or net game activities). Cochran's Q and the $I^{2}$ Index tests were used to assess statistical heterogeneity. For the $I^{2}$ Index; 0-40\% may represent low heterogeneity; $30-60 \%$ moderate heterogeneity; $50-90 \%$ substantial heterogeneity; and $75-100 \%$ considerable heterogeneity [14]. The moderator meta-analysis that examined the method of measuring MVPA was also used to examine methodological heterogeneity.

\section{Results}

\section{Description of the studies}

The stages of the systematic review and study exclusions are shown Fig. 1. The initial database search returned
8,300 journal articles prior to de-duplication, or 5,132 journal articles once duplicates were removed. Following title and abstract review, 71 full text articles were retrieved and assessed for eligibility. Twenty-eight papers representing 25 studies (7 middle school and 18 high school studies) met all inclusion criteria and were included in the systematic review. All study selection discrepancies between the two reviewers were resolved through discussion and the third reviewer was not consulted. Missing data were requested from 13 study authors to determine study eligibility and/or to obtain sufficient data for the study to be pooled into the metaanalysis. Nine authors responded to the email communication, of which five provided the additional requested data. No additional eligible articles were retrieved from the reference lists of the included articles.

\section{Characteristics of included studies}

The characteristics and outcomes of the studies are shown in Tables 1 and 2. Publication dates ranged from 2005 [19-23] to 2014 [24, 25]. The studies were primarily conducted in the United States of America (USA; $n=13)$ and Australia $(n=6)$, with two studies in Portugal and one each in the United Kingdom, Poland, Brazil and Hong Kong. All seven middle school studies were conducted in the USA. Almost $50 \%$ of the studies were of cross-sectional design $(n=12)$, followed by RCT's $(n=4)$, non-RCT's $(n=3)$, pre-post

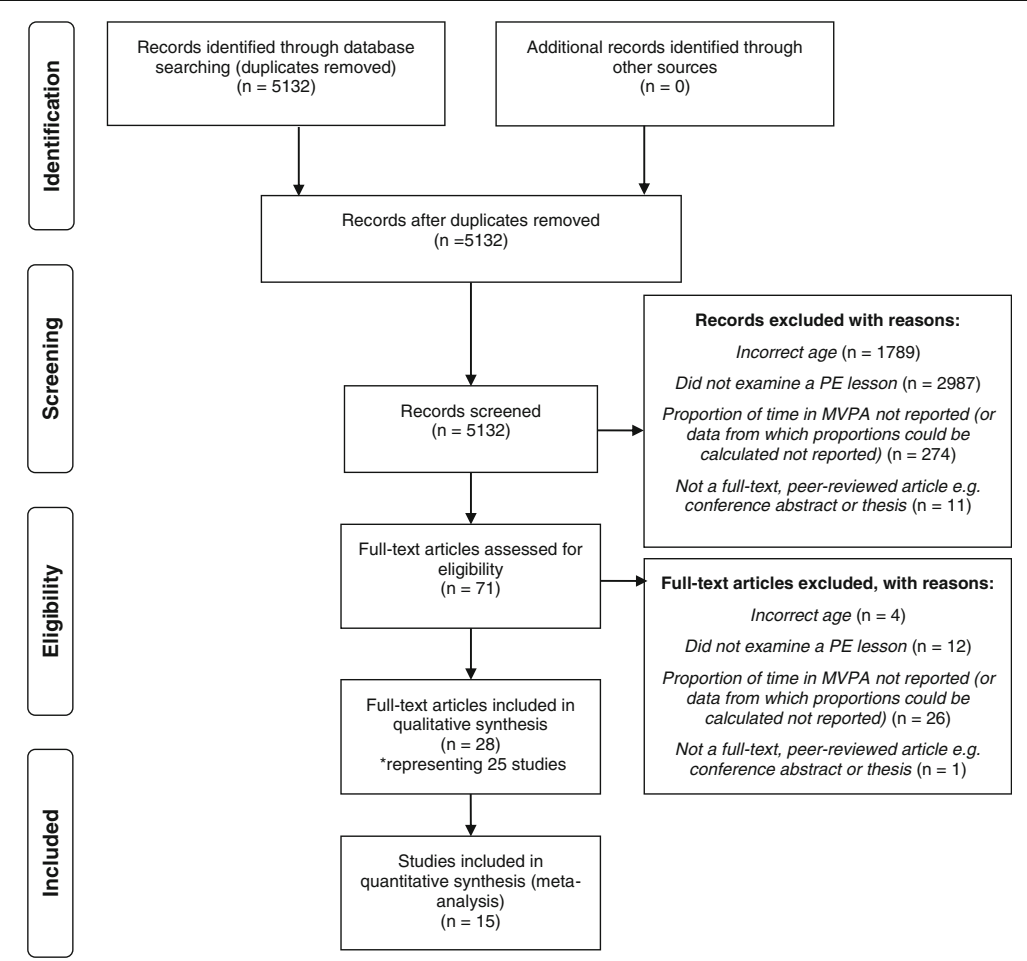

Fig. 1 PRISMA flow chart illustrating study inclusions through the stages of the systematic review and meta-analysis 


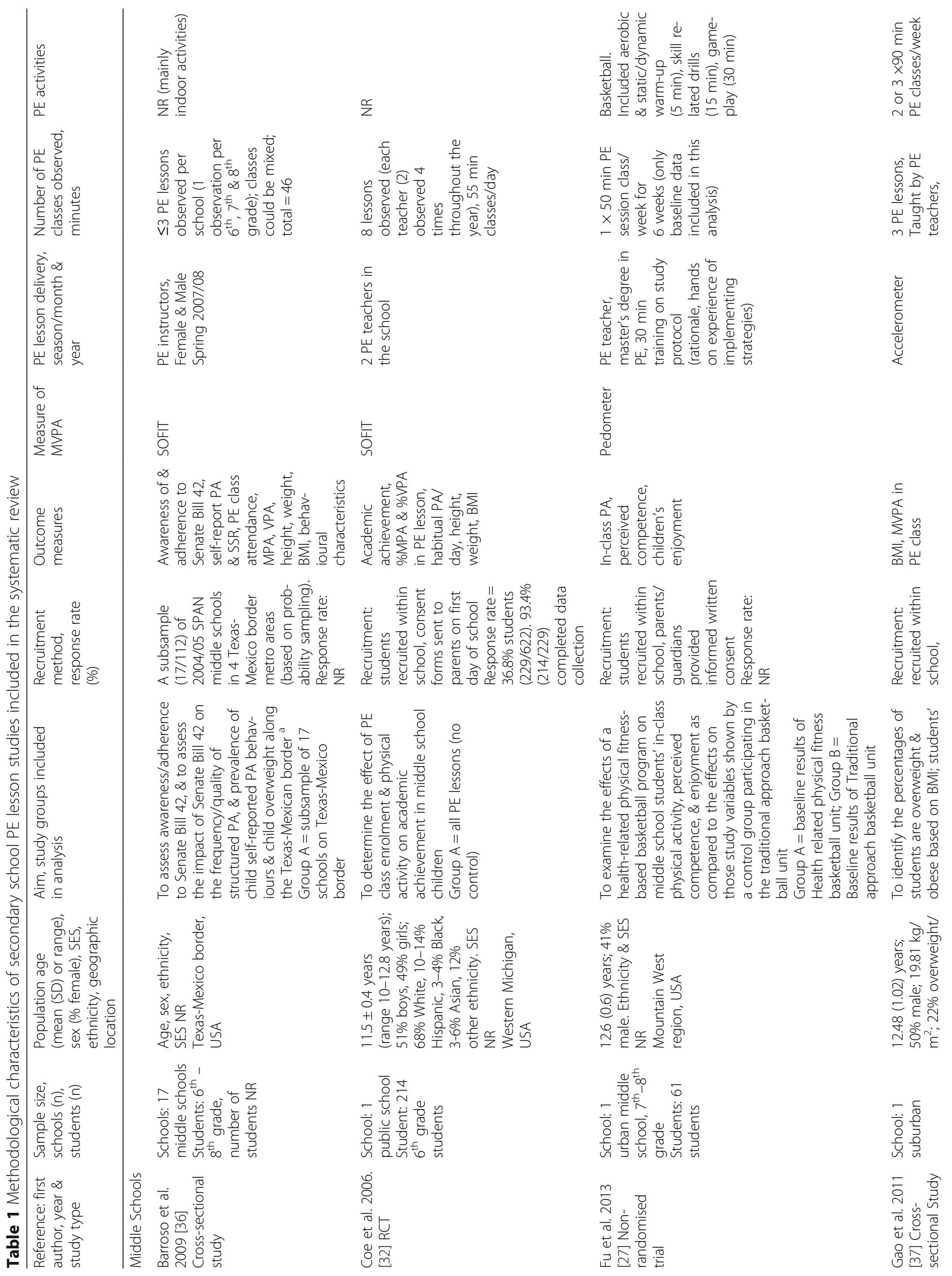




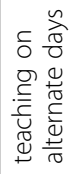

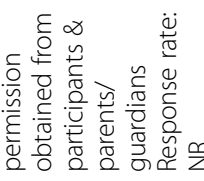

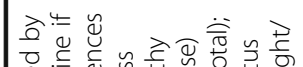

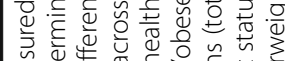

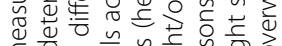

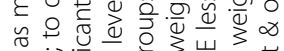

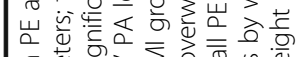

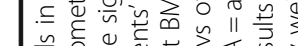

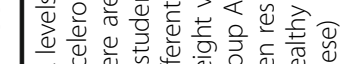

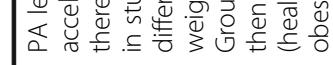

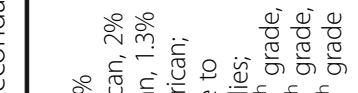

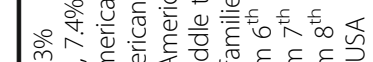

O.

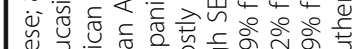

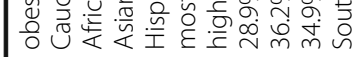

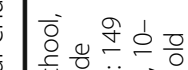

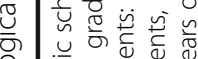

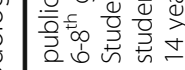

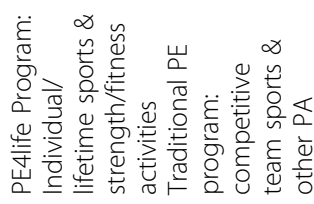

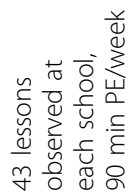

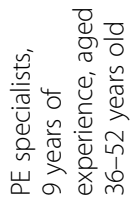

氙

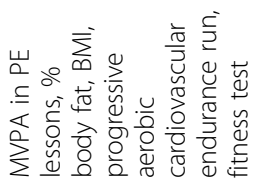

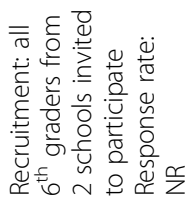

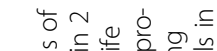

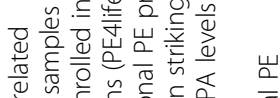

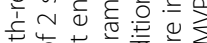

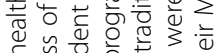

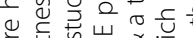

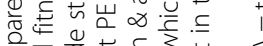

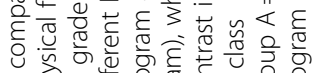

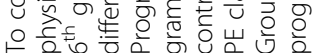

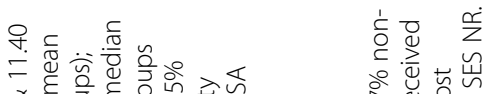

ळ

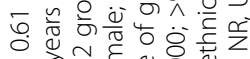

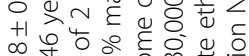

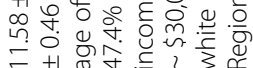

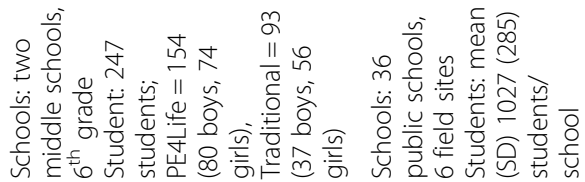

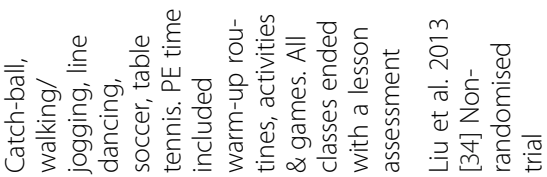

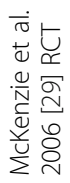

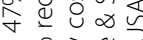

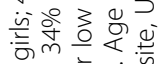

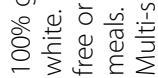

$\frac{\Upsilon 2}{z}$

\section{능}

苞㤩

这 $\overline{-1}$

古

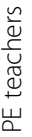

농

近

충 중

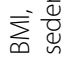

๖े $\frac{u}{\mathrm{O}}$

这

है $\frac{0}{0}$

는

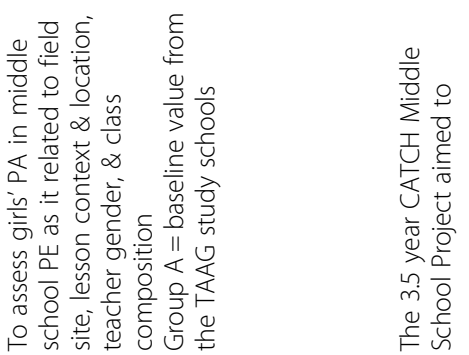

f

$\frac{\varrho}{\circ}$

है

类

$\stackrel{\frac{0}{\frac{0}{0}}}{\frac{0}{\varepsilon}}$

这葆

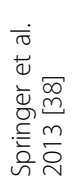




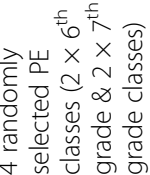

\section{岁

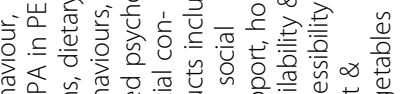

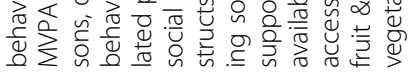

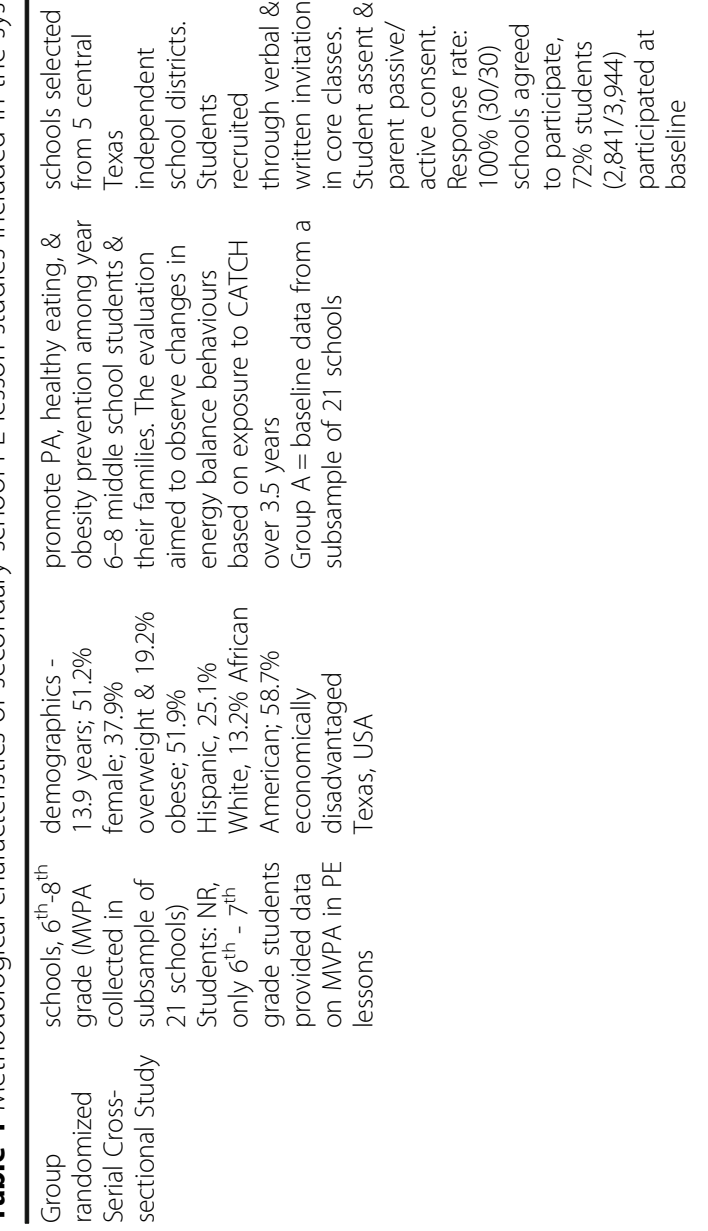

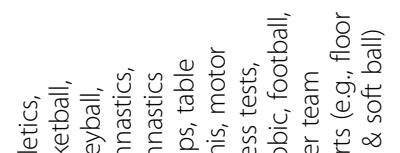

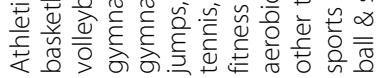

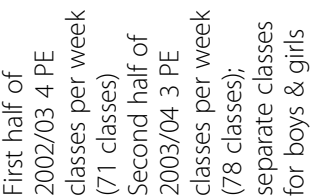

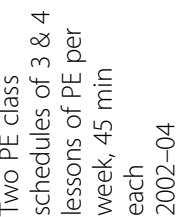

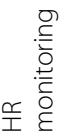

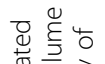

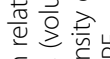

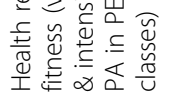
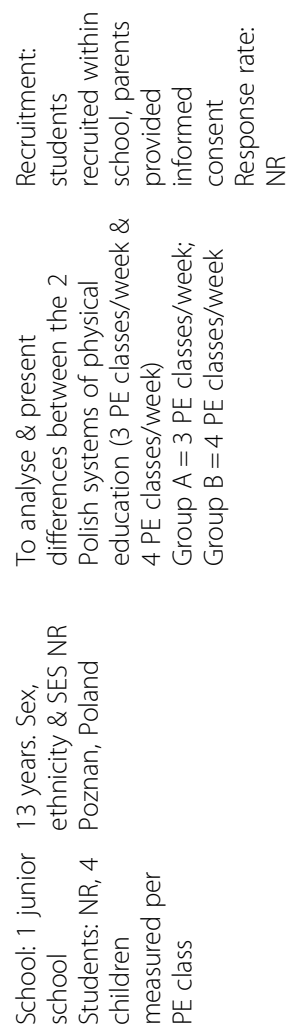

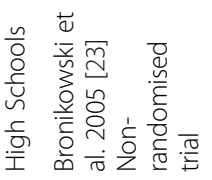

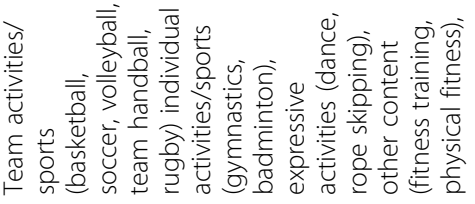

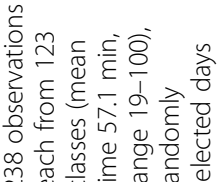

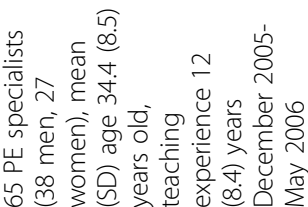

눙

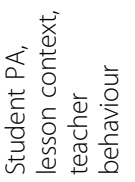

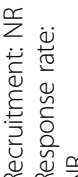

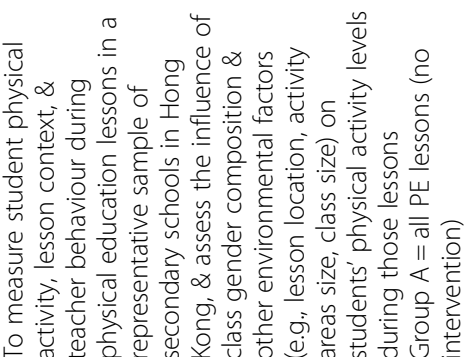

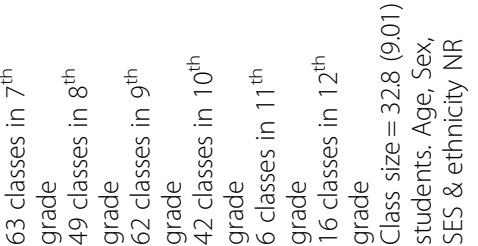

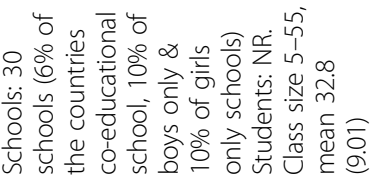

䎡写突

文这岕总 


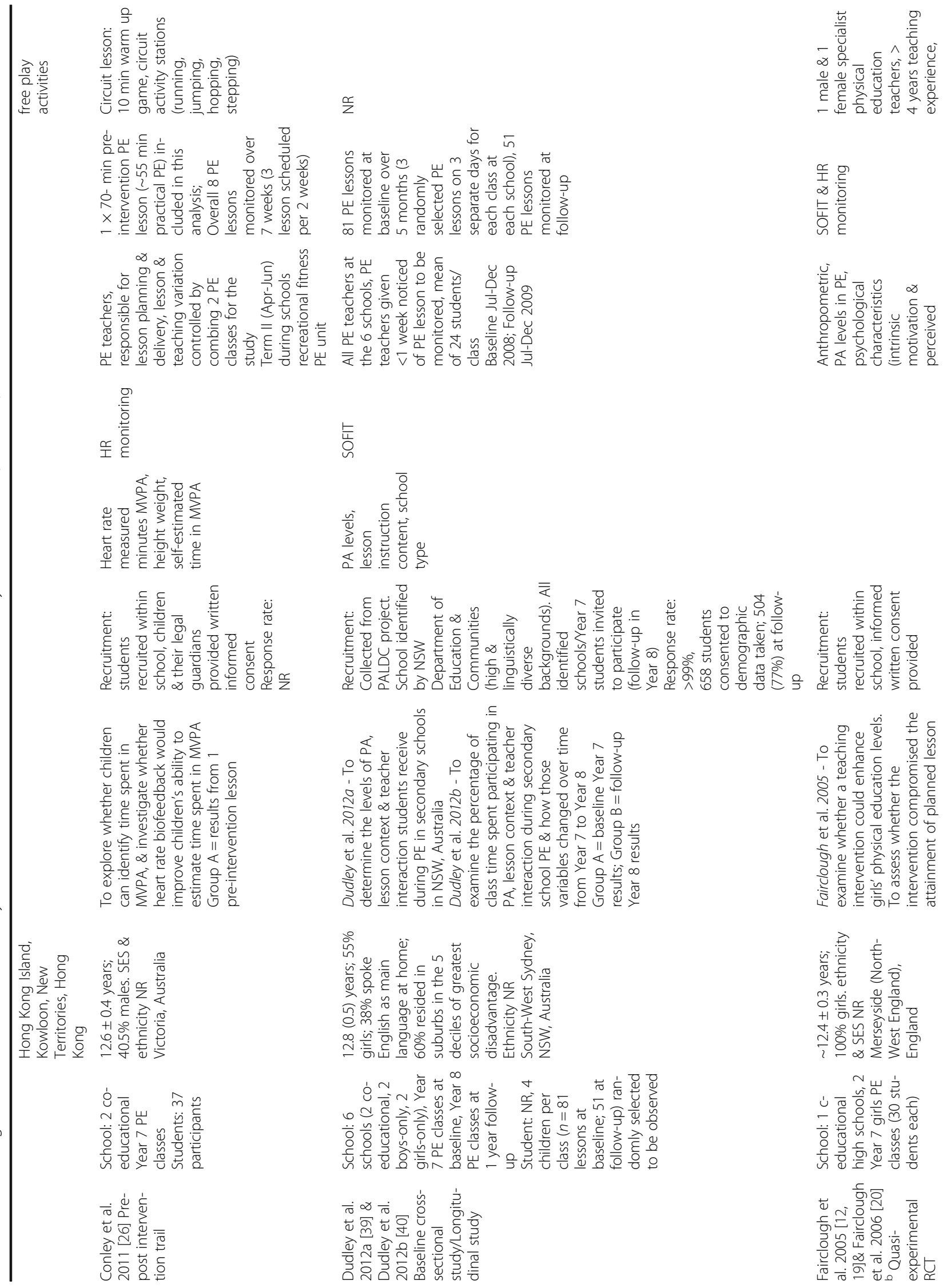


응

$\frac{\sim}{\mathbb{0}} \frac{\pi}{\cup}$

突

$+$

ฮิ

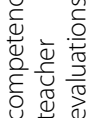

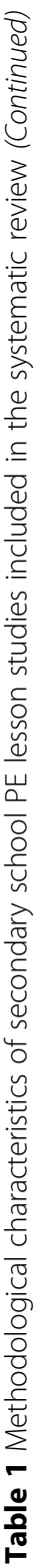

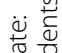

出

등응

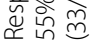

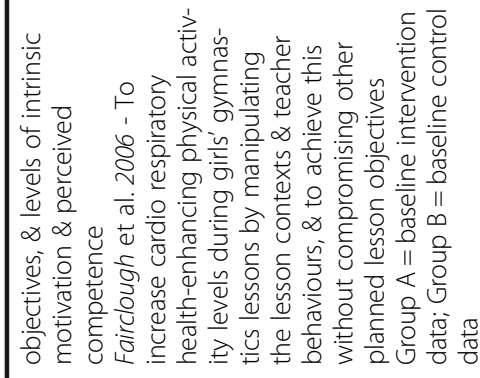

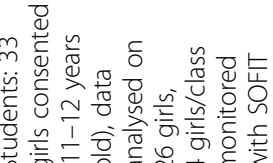

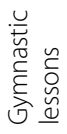

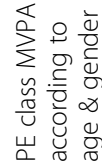

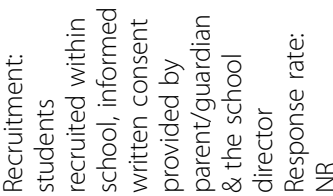

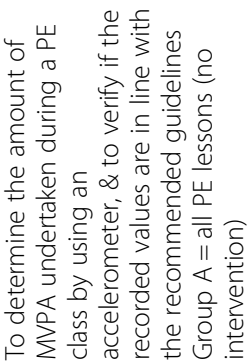

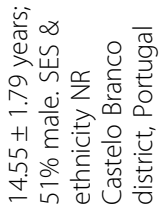

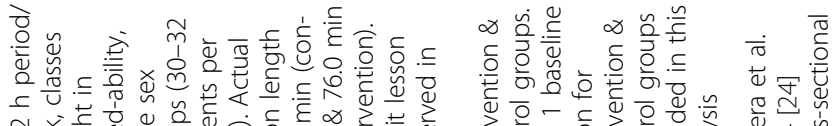

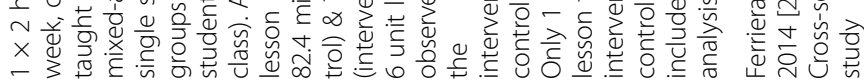




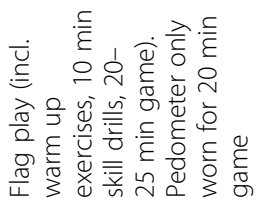

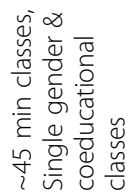

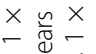

ज政

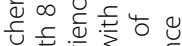

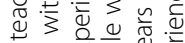

山己

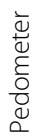

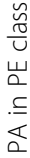

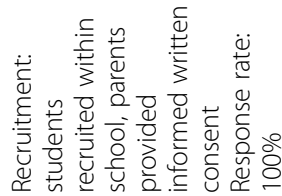

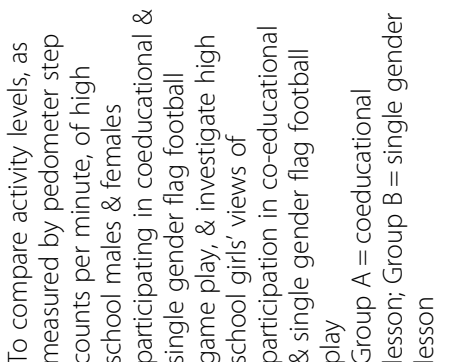

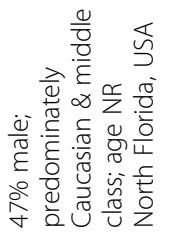

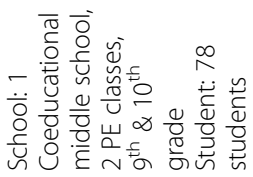

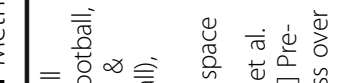

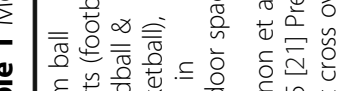

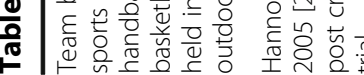

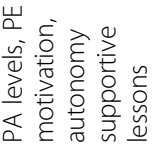

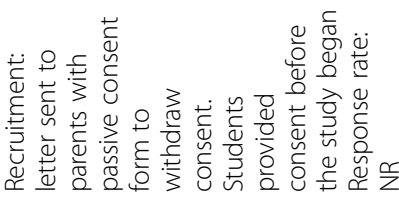
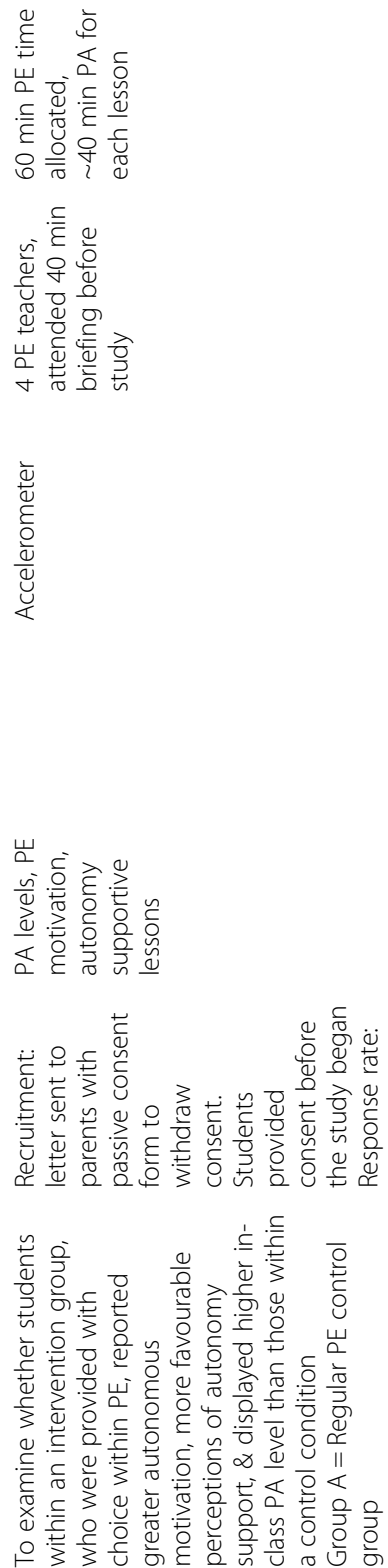

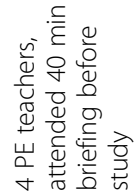

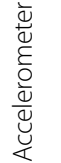

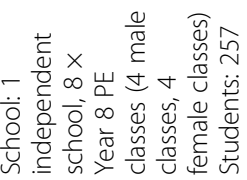

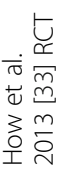

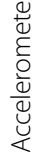

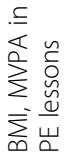

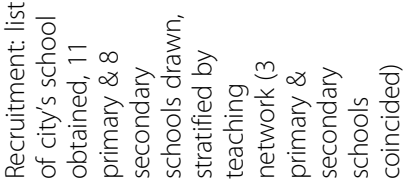

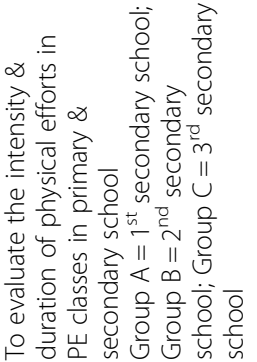

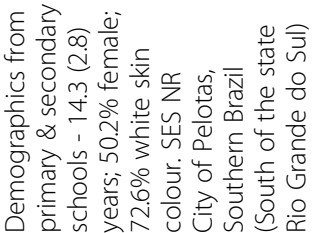

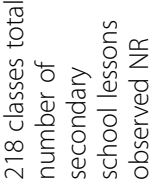

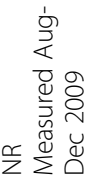$$
\text { (n) }
$$

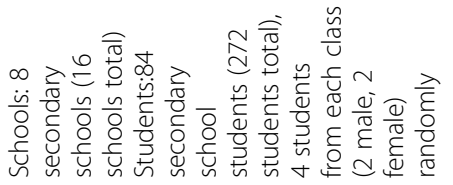

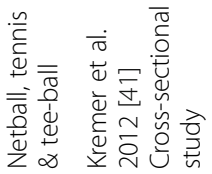




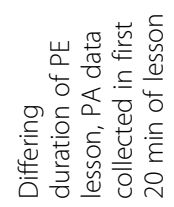

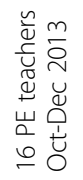

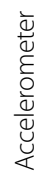

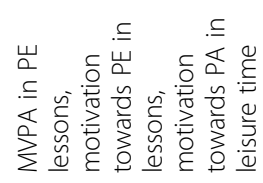

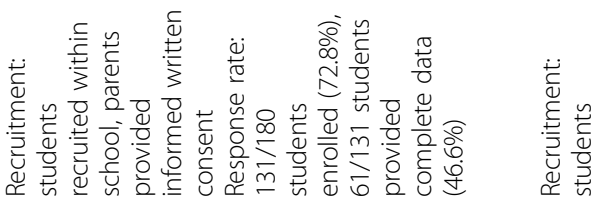
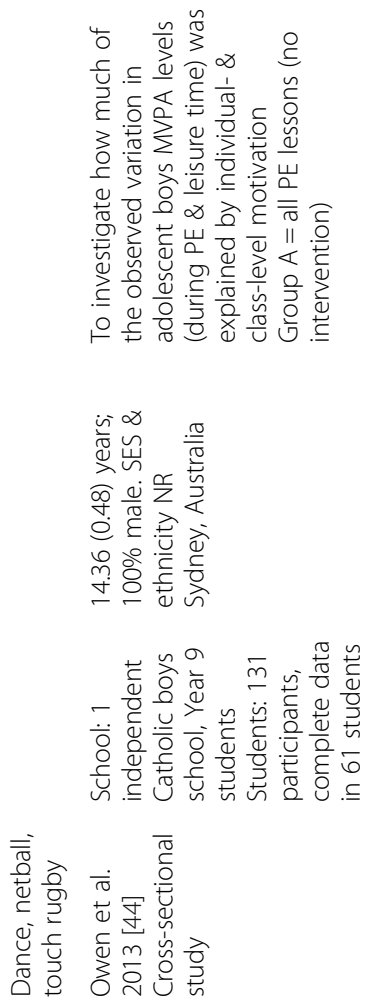

i⿱宀 犬㔾

कें हैं

ฮ요

in

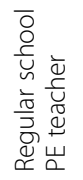

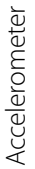

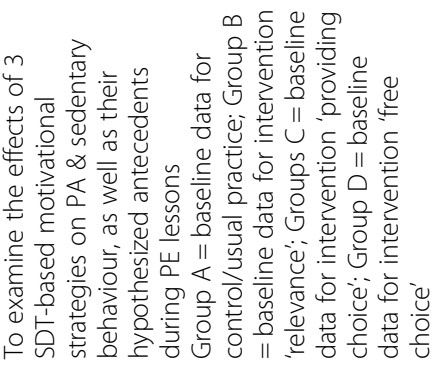

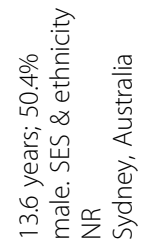

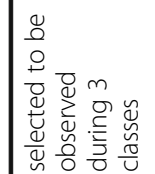

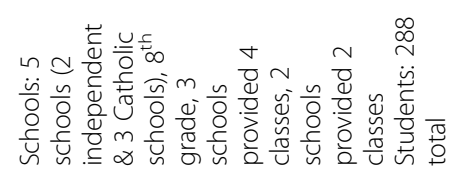
$\dot{\vec{\sigma}} \stackrel{\infty}{\sigma} \ddot{\mathrm{J}}$
a

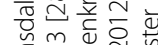
品 


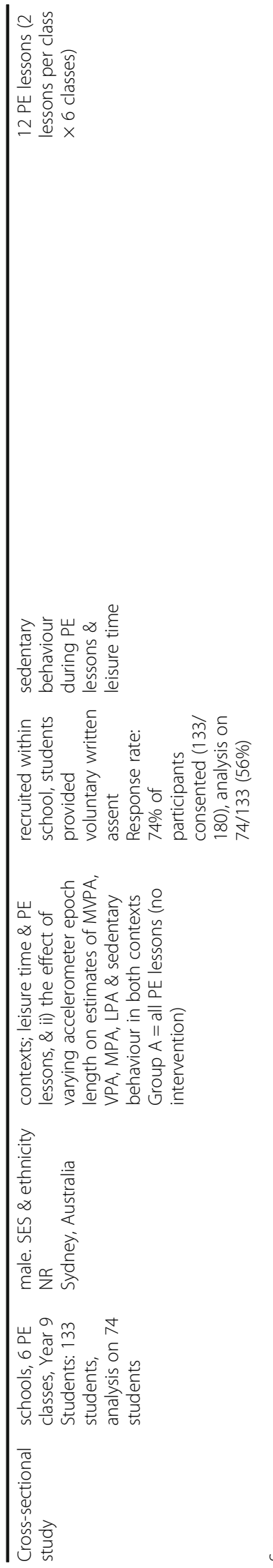

$\frac{\Upsilon}{z}$

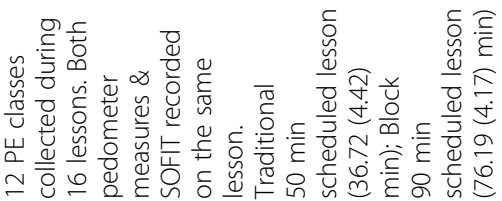

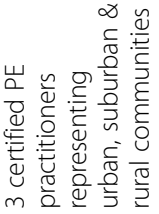

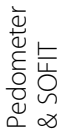

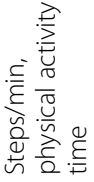

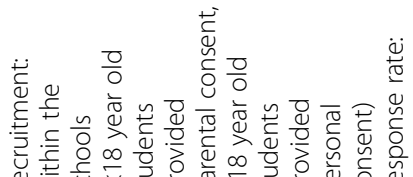

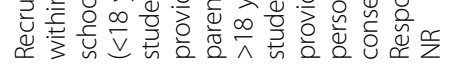

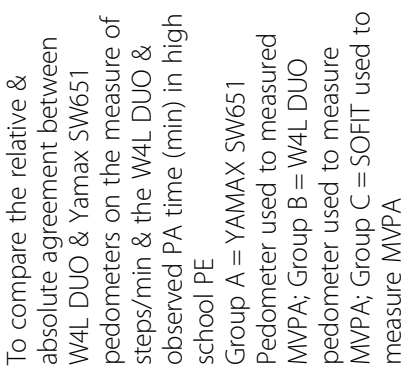

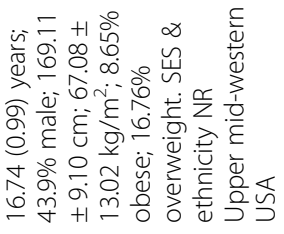

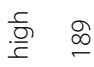

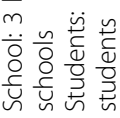

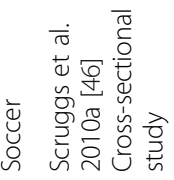

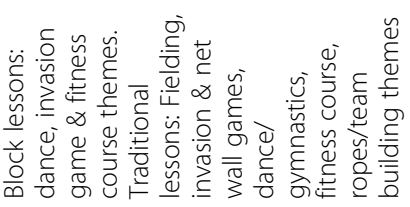

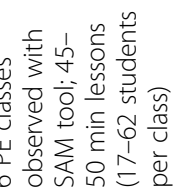

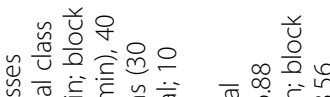

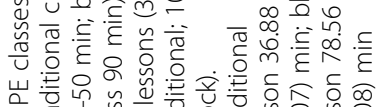

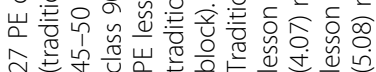

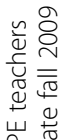

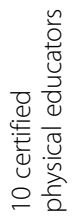

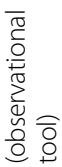

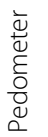

$\underset{\substack{\infty \\ \vdots}}{\infty}$

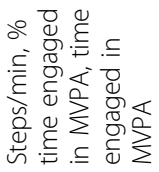

뜸

至管

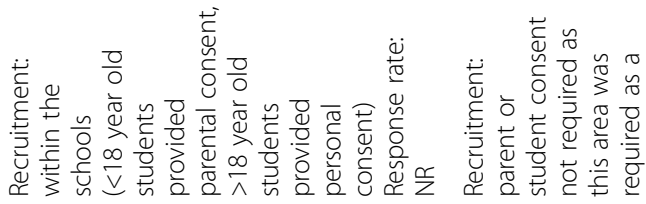

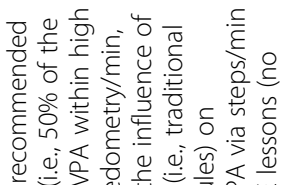

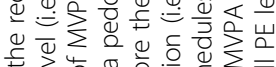

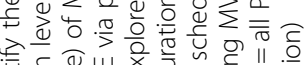

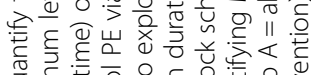

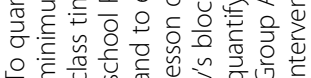

줄 范

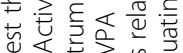

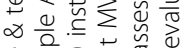

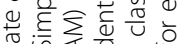

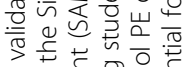

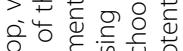

O

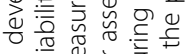

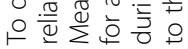

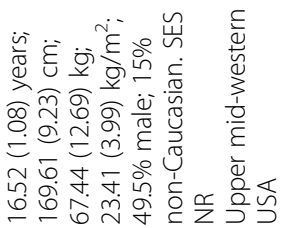

mి $\stackrel{0}{\stackrel{0}{0}}$

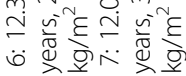

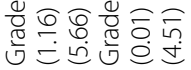

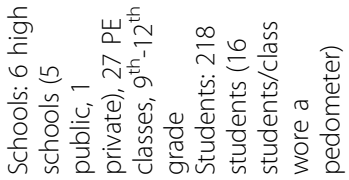

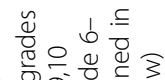

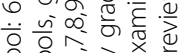

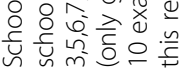

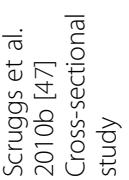

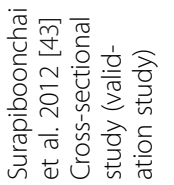




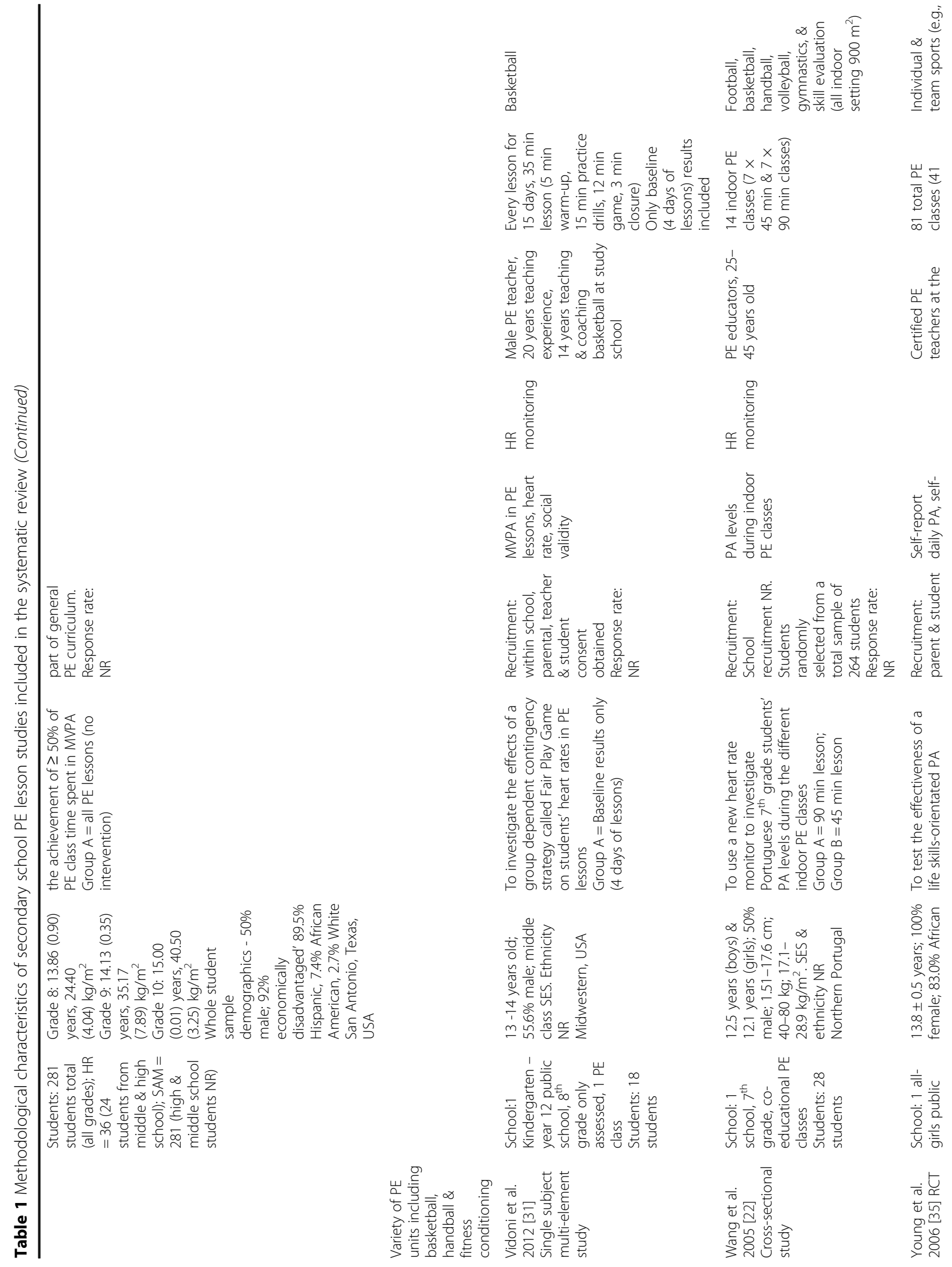




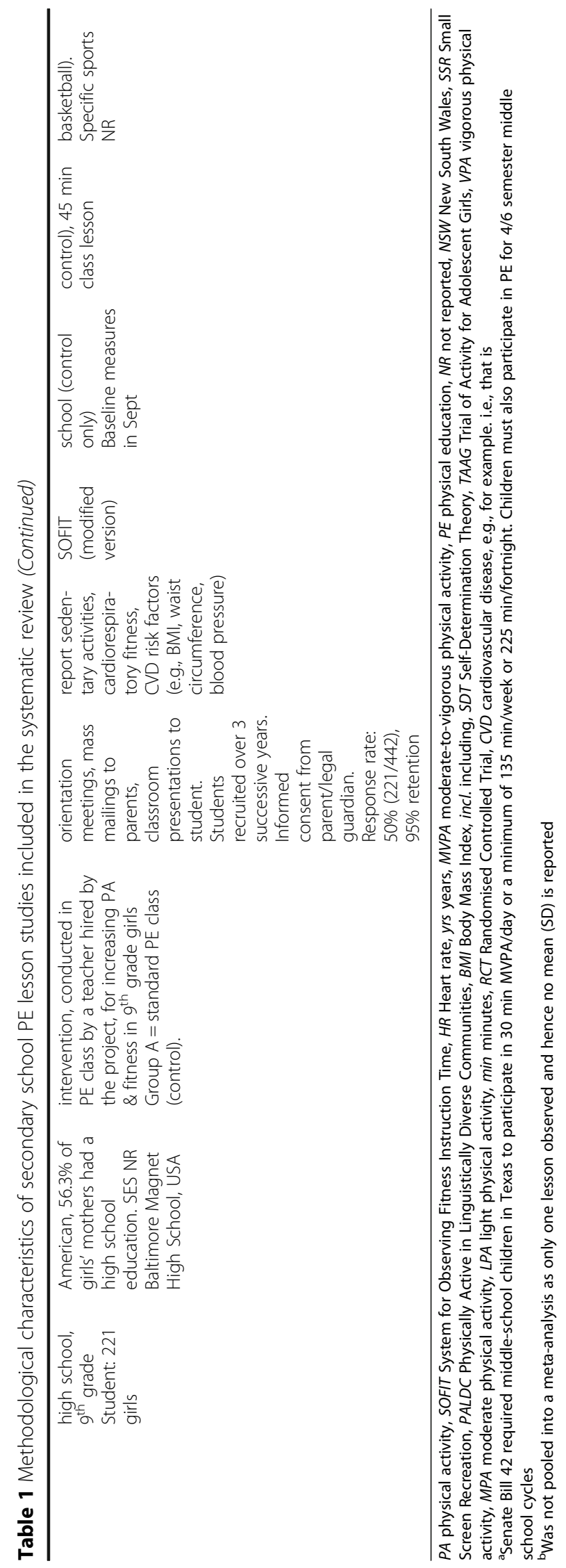




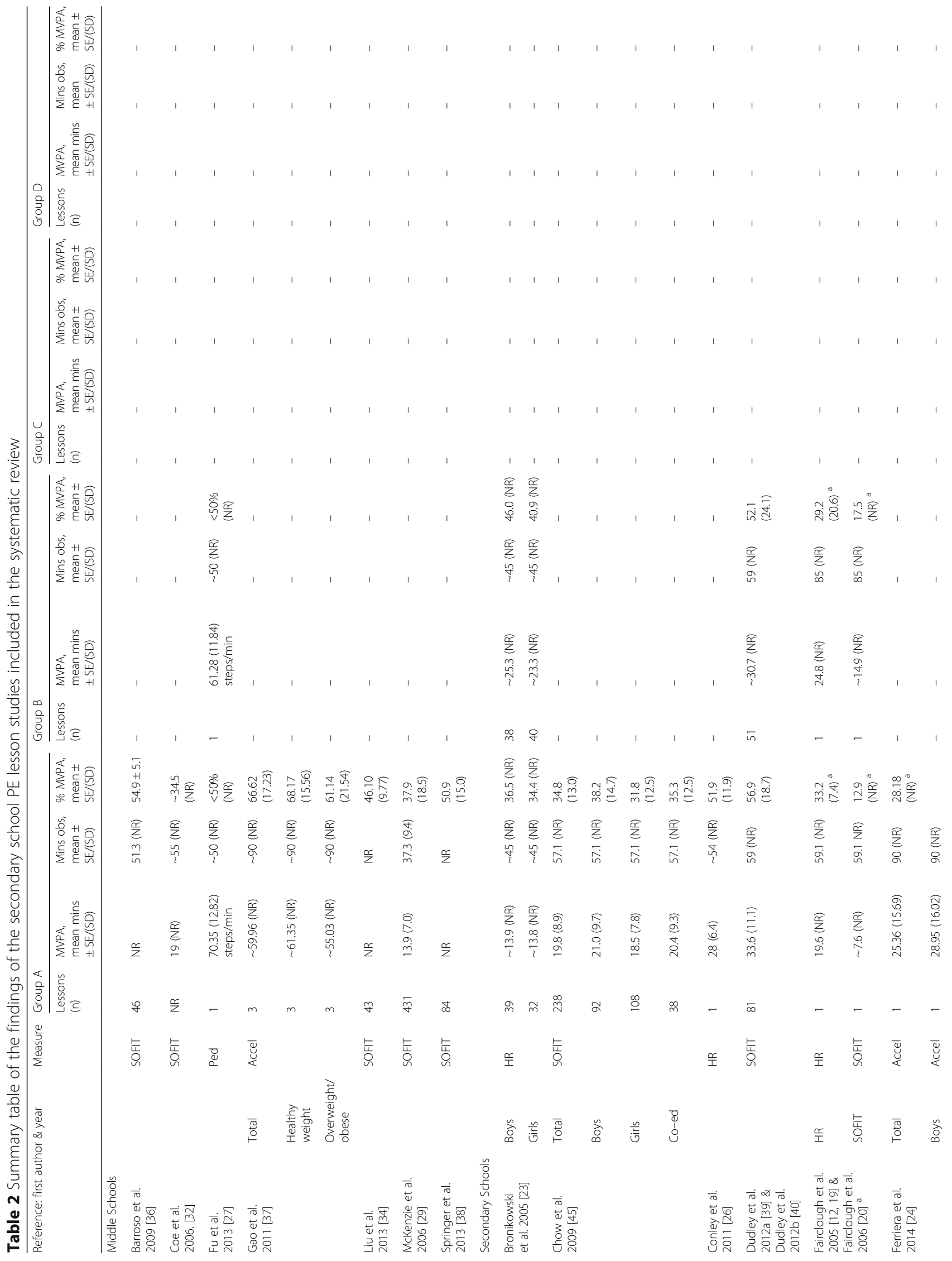




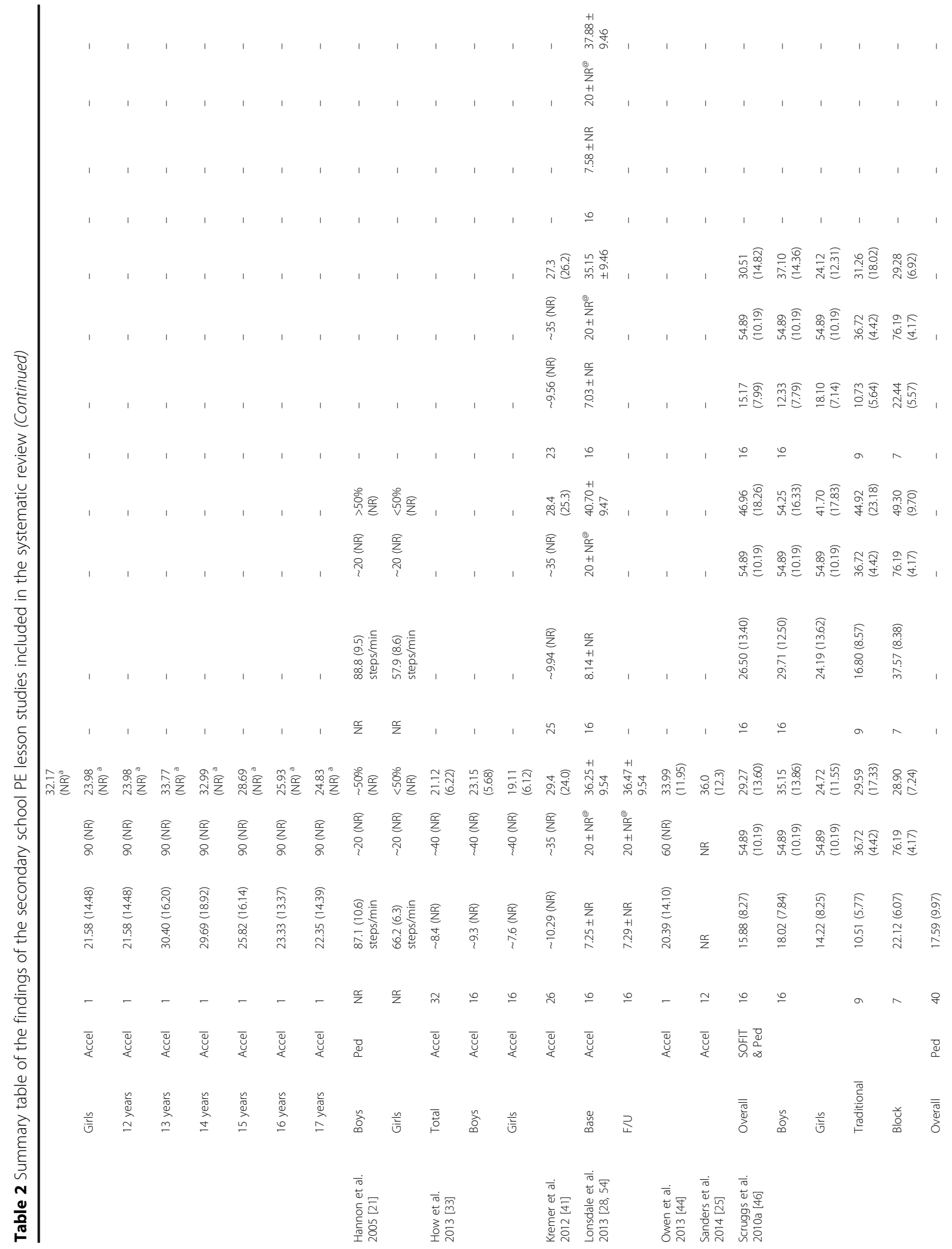




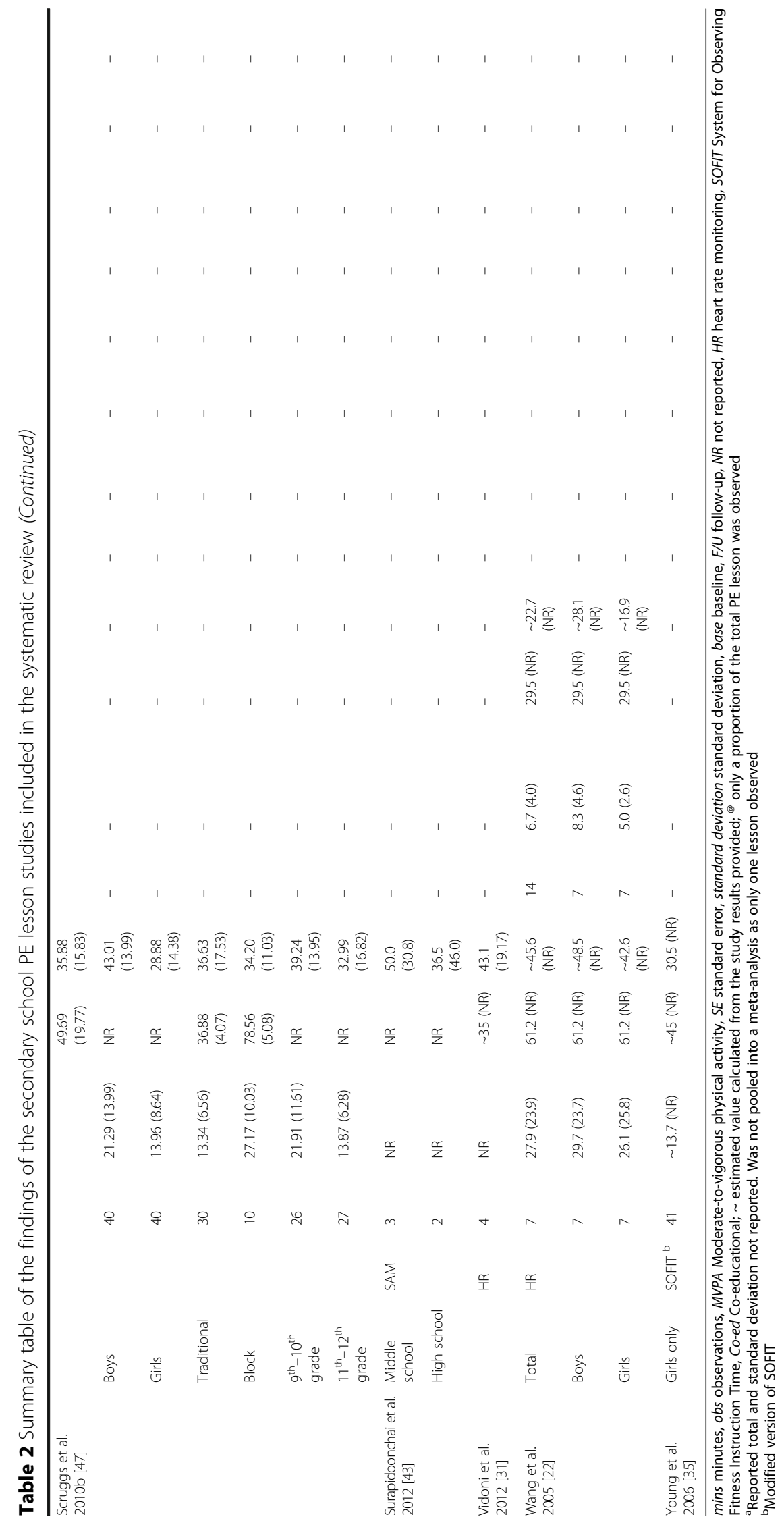


studies $(n=2)$, quasi-experimental $\operatorname{RCT}(n=1)$, cluster RCT $(n=1)$, single subject multi-element study $(n=1)$ and a group randomised serial cross-sectional study $(n=1)$. Of the 12 studies that were not of cross-sectional nature, six studies contributed baseline intervention and/or control group data to the systematic review $[19,20,26-31]$ and the remaining six studies contributed only control group data at follow-up [21, 23, 32-35].

The eligible studies were conducted in 88 middle schools and 77 high schools. Six of the seven middle school studies reported data on both male and female students and did not separate results by sex [27, 32, 34, 36-38] and one study reported a female only sample [29]. Six of the 18 high school studies [26, 30, 31, 39-43] reported data on both male and female students and did not separate results by sex. Of the remaining 12 high school studies, two were conducted with a female only sample $[19,20,35]$, two with a male only sample [25, 44], and eight examined both male and female students and reported results separately for each sex [21-24, 33, 45-47].

Twelve studies measured MVPA through observational measures (e.g., SOFIT) [19, 20, 29, 32, 34-36, 38$40,43,45-47]$, seven used accelerometers [24, 25, 28, $33,37,41,44]$, five used heart rate monitors $[19,20,22$, $23,26,31]$ and four used pedometers [21, 27, 46, 47]. The majority of middle school studies assessed physical activity through observational measures $(n=5 / 7)$. Studies in a high school setting used a range of measurement tools including observation methods $(n=7)$, accelerometry $(n=6)$, heart rate monitoring $(n=5)$, and pedometers $(n=3)$. Three high school studies used two methods of PE lesson monitoring [19, 20, 46, 47].

The number of PE lessons observed in each study ranged from 1-431. In total, more than 609 middle school PE lessons and 837 high school PE lessons were monitored. One middle school study [32] and one high school study [21] did not report number of lessons monitored. Lesson length was highly variable in both middle school (range: 37-90 $\mathrm{min} /$ lesson) and high schools (range: 20-90 min/lesson). All middle school studies $(n=7 / 7)$ and the majority of high school studies $(n=15 / 18)$ monitored PE lessons that were led by PE teachers, specialists or instructors. The remaining studies did not state who led the PE lesson [23, 41, 44]. The random effects model was used for main and moderator meta-analyses as there was heterogeneity among the studies (main meta-analysis: $\mathrm{Q}=455.8, \mathrm{df}=14, p<0.001$, $\mathrm{I}^{2}=96.9 \%$; moderator analysis by school level: middle school $\mathrm{Q}=70.6, \quad \mathrm{df}=4, \quad p<0.001, \mathrm{I}^{2}=94.3 \%$, high school $\mathrm{Q}=274.4$, $\mathrm{df}=9, p<0.001, \mathrm{I}^{2}=96.7 \%$; moderator analysis by type of physical activity measurement: observational methods $\mathrm{Q}=183.7, \mathrm{df}=7, p<0.001, \mathrm{I}^{2}=$ 96.2\%, accelerometer methods $\mathrm{Q}=49.1, \mathrm{df}=4, p<0.001$, $\left.\mathrm{I}^{2}=91.9 \%\right)$.

\section{Risk of bias}

The risk of bias coding criteria and results of the appraisal are outlined in Table 3. The representativeness of the school, class and student sample were the primary sources of potential bias. Few studies demonstrated that i) the schools examined were representative of other schools $(n=5)$, ii) the classes chosen to be monitored were representative of all classes $(n=9)$, or iii) the students chosen to be monitored were representative of the population $(n=12)$. All studies adequately described the demographic characteristics of the school sample. The majority of studies used an objective measure of physical activity or cited validation studies $(n=22)$ and stated reliability data $(n=22)$.

\section{MVPA in secondary school PE lessons}

Of the 25 studies included in the systematic review, the percentage of PE lesson time spent in MVPA ranged between 12.9 and $68.2 \%$. Fifteen studies provided the necessary data to be pooled into a meta-analysis. The pooled analysis of these studies (Fig. 2) showed that the mean $(95 \% \mathrm{CI})$ percentage of $\mathrm{PE}$ lesson time that secondary school students spent in MVPA was 40.5\% (34.8-46.2\%).

\section{Moderator analyses}

- Student participation in MVPA during PE by school level (middle vs high school)

Of the 15 studies included in the meta-analysis, five were conducted in middle schools and 10 in high schools. Middle school students spent a mean (95\%CI) of $48.6 \%(41.3-55.9 \%)$ of PE lesson time in MVPA in comparison to $35.9 \%$ (28.3-43.6\%) of PE lesson time spent in MVPA by high school students (Fig. 3).

- Student participation in MVPA during PE by physical activity measurement type Five of the 15 studies in the meta-analysis assessed MVPA with accelerometers, nine used observational methods, one used heart rate monitors, and one used pedometers. One study used both observational measures and pedometers. For studies using accelerometers, students spent 34.7\% (25.1-44.4\%) of PE lesson time in MVPA (Fig. 4); in comparison to $44.4 \%$ (38.3-50.5\%), 43.1\% (24.3-61.9\%) and $35.9 \%$ (31.0-40.8\%) when measured using observational methods, heart rate monitors and pedometers, respectively.

- Student participation in MVPA during PE by PE lesson type

An analysis to examine whether MVPA in lesson time differed by lesson type was not conducted due to a lack of detailed information provided on PE activities. While nine of the 15 studies included in 


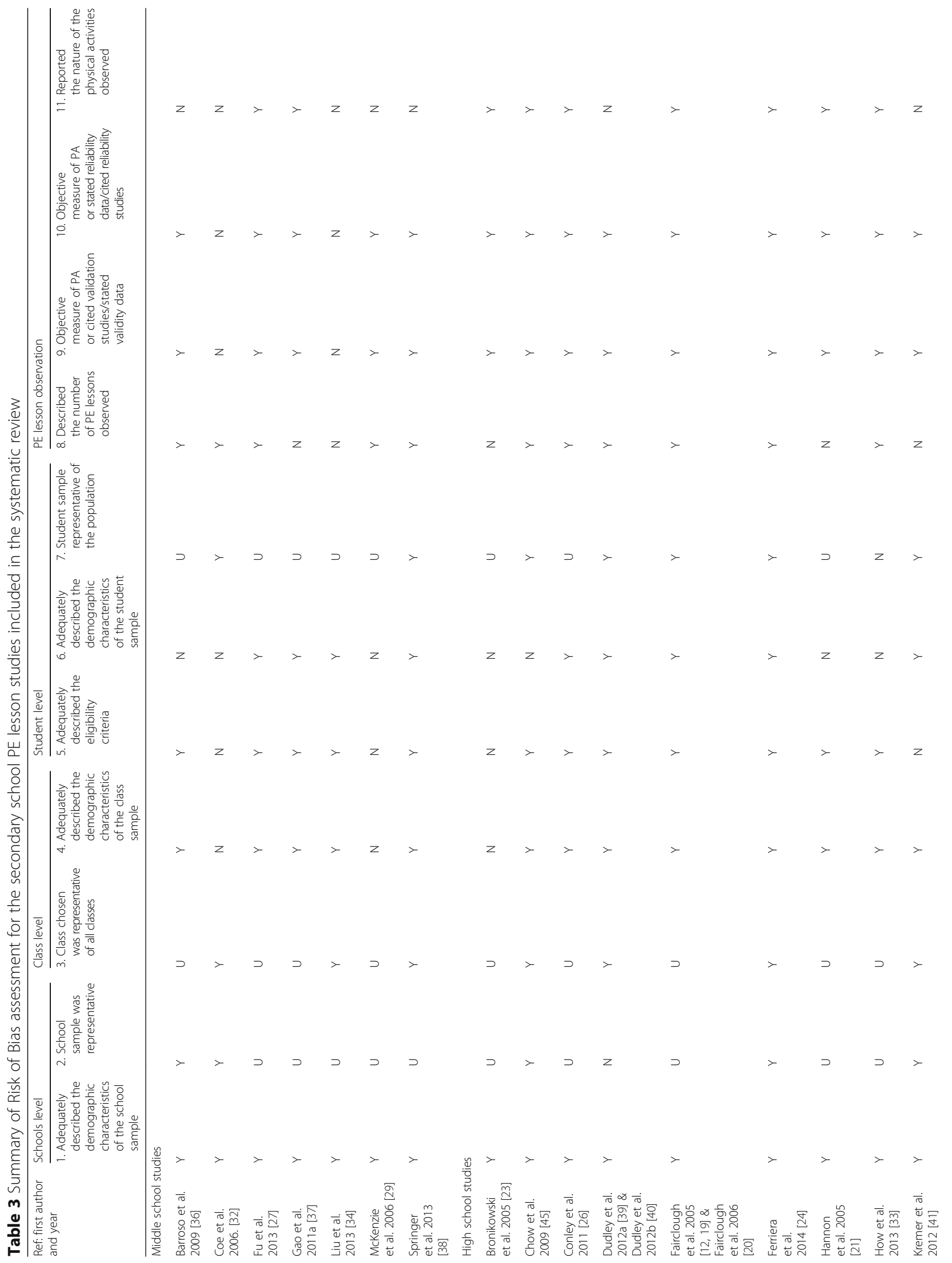




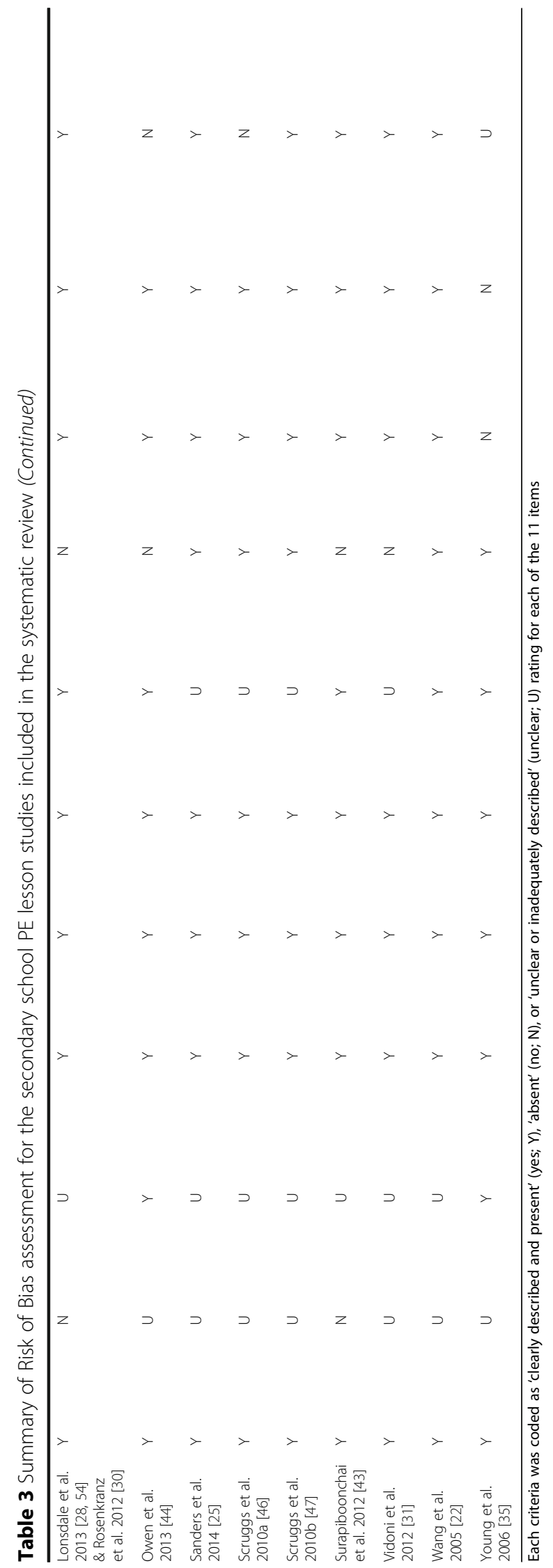




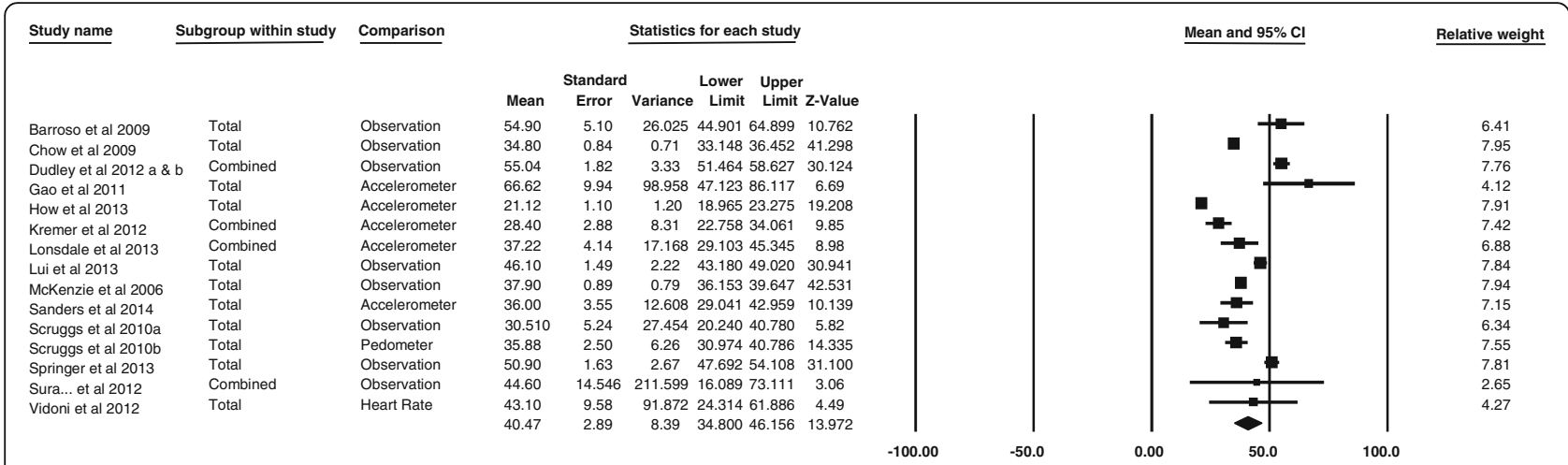

Fig. 2 Individual study and pooled results of the percentage of secondary school PE lesson time spent in MVPA

the meta-analysis provided details on the type/s of physical activity that students were engaged in, the majority (7/9 studies) reported on lessons that included activities that fell into multiple categories (fitness orientated activities, team invasion games, dance and gymnastics or net game activities) and the proportion of time spent in MVPA was not reported separately for each lesson type.

\section{Discussion}

\section{Summary of the evidence}

This systematic review and meta-analysis aimed to determine the proportion of secondary (middle and high) school PE lesson time that students spend in MVPA. Of the 25 studies found, the proportion of time spent in MVPA varied considerably between 12.9 and $68.2 \%$ of lessons. The meta-analysis of 15 studies found that the proportion of lesson time spent in MVPA is typically below the US CDC [9] and UK AfPE [11] recommendation of $50 \%$ of $\mathrm{PE}$ lesson time, with only $40.5 \%$ of secondary school lesson time spent in MVPA. Middle school students were found to spend a higher proportion of lesson time in MVPA (48.6\%) in comparison to high school students (35.9\%), although there was considerable variability in the proportions from both groups. Results differed according to the method of MVPA assessment, ranging from $34.7 \%$ in accelerometer-assessed lessons to $44.4 \%$ for observational-assessed studies. Caution should be taken when interpreting these finding as the confidence intervals for the moderator analyses overlapped and only one study reported findings using heart rate monitors and pedometers. The heterogeneity in study findings may be due to the different ages of the students and the different types of activities in PE (which were often not reported). The studies also differed in their measurement protocols, such as using a variety of measurement instruments (e.g., accelerometers vs SOFIT) with different definitions of reporting time (e.g., scheduled lesson vs actual lesson length), which may have also contributed to the heterogeneity in results between the studies. We were unable to explore differences in MVPA according to lesson type due to a lack of detailed information and data provided on PE activities.

The findings of the overall proportion of MVPA in PE lessons $(40.5 \%)$ are broadly similar to the previous secondary school review on this topic published in 2005 [12] which reported the proportion of PE lesson time

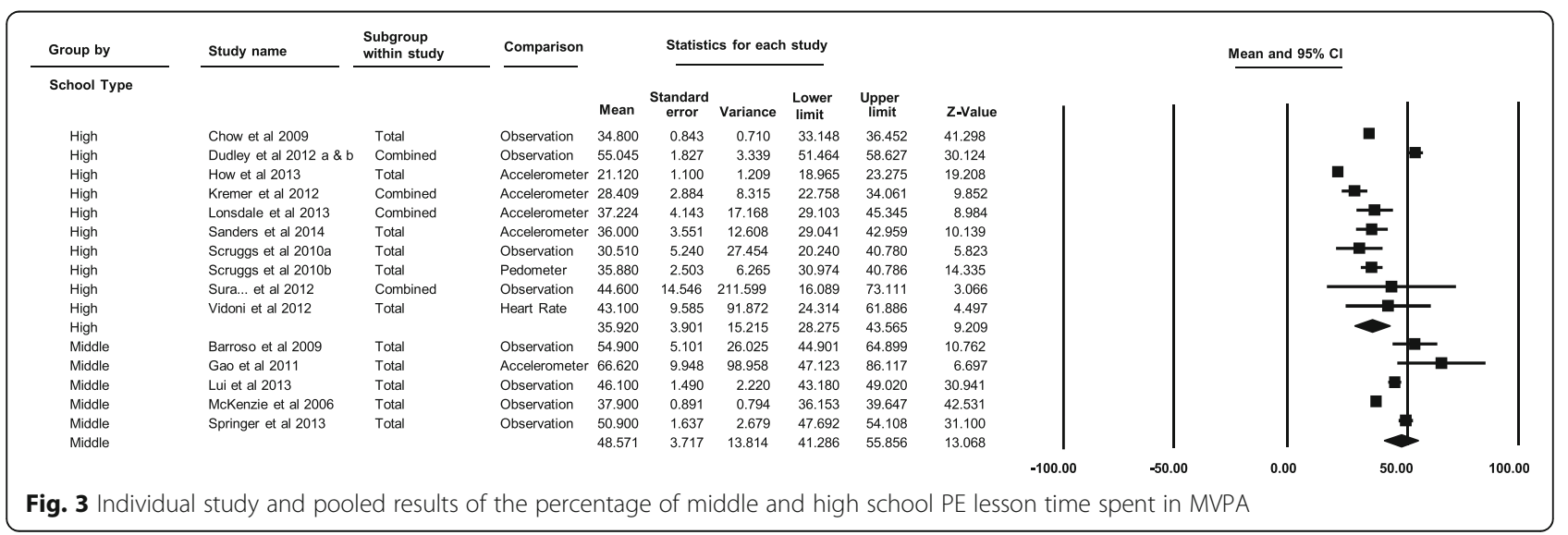




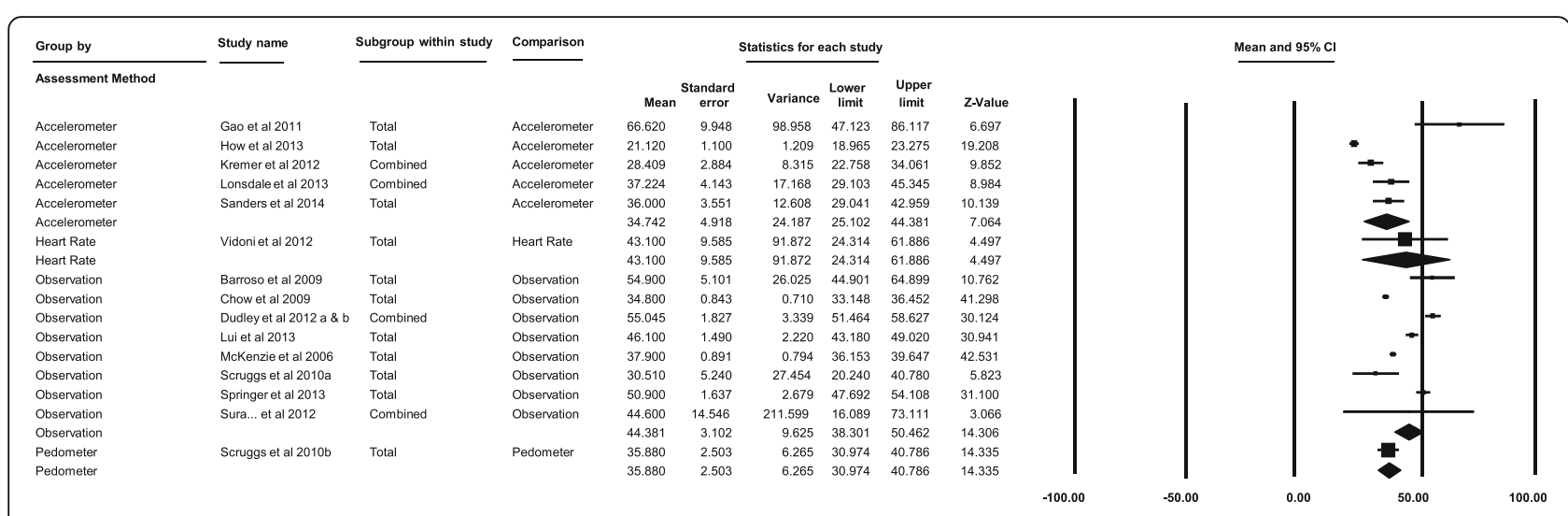

Fig. 4 Subgroup meta-analysis of the percentage of secondary school PE lesson time spent in MVPA according to measurement method (accelerometers, heart rate monitors, observational methods and pedometers)

spent in MVPA separately according to the type of measurement instrument used, ranging between $27-47 \%$ of the lesson. There were variations in findings between the two reviews according to the type of measurement instrument used. For example, accelerometer-measured lessons reported the lowest levels of MVPA in the present review (34.7\%) and the highest proportion MVPA in the previous review (46.8\%) [12]. The variation in findings may be explained by differences in methodology between the two reviews; the 2005 review was not a systematic review, did not assess risk of bias and a meta-analysis was not conducted so we are unable to comment on any biases of the component studies which may have influenced the results. The proportion of studies assessed using different measurement instruments also varied between the two reviews, which may have influenced the findings since different instruments may be associated with different types of bias. For example, of the 15 studies included in our meta-analysis, 9 studies used observational measures. Whilst this is a similar number as in the previous review, it forms a higher proportion of the studies (60\% v's $25 \%$ ). Methodological inconsistencies between the measurement instruments (e.g., length of the monitored PE lesson and the measurement of different elements of activity to calculate MVPA) make it difficult to draw firm conclusions. The implications of methodological inconsistencies and potential biases are discussed in more detail below. Regardless of the type of measurement method used, the findings suggest that overall little progress has been made in engaging adolescents in more MVPA during PE lessons.

Some differences in MVPA levels were observed between studies in the middle and high school setting. The moderator analyses found that, on average, students in middle school PE lessons were observed to be almost meeting the US CDC [9] and UK AfPE [11] recommendation with $48.6 \%(41.3-55.9 \%)$ of lesson time being spent in MVPA, compared with 35.9\% (28.3-43.6\%) of PE lesson time in high schools. In a recent metaanalysis on MVPA in elementary school PE lessons using the same methodology [17], elementary school students were found to engage in MVPA for 44.8\% (28.2-61.4\%) of the PE lesson. As the confidence intervals for the elementary, middle and high school analyses overlap, the findings suggest that the level of MVPA in PE across each school setting are relatively comparable. Caution should be taken in interpreting the school level specific results as it is unclear whether the seeming decline in MVPA in PE lessons from middle school to high school was a result of the type of measurement instruments used rather than the school setting. For example, four of the five (80\%) middle school studies included in the meta-analysis assessed MVPA using observational methods, in comparison to four of ten (40\%) high school studies. The previous review did not examine MVPA in middle and high school PE lessons separately, so we are unable to comment on progress in the different secondary school settings.

As all middle school studies were conducted in the USA, the generalisability of the middle school findings to other countries is unclear. Middle schools in the USA normally enrol students aged between 11-13 years old $\left(6^{\text {th }}-8^{\text {th }}\right.$ grade), although this can vary depending on school districts. This age range spans both elementary and high school ages in other countries such as the UK and Australia. Further research reviewing MVPA in middle school lessons outside of the USA is needed to examine whether the observed level of MVPA in middle school PE lessons is uniform or isolated to the USA. Gaining a better understanding of the strategies implemented to build active lessons in middle school PE lessons in the USA may provide insight to build more active lessons in high schools. Continuing to intervene within the middle school setting remains important for both maintaining activity levels and ensuring activity is undertaken equally across the student population. 
Barriers to delivering more active PE lessons have been described as institutional (e.g., school policies, a crowded curriculum, limited facilities and equipment, and insufficient departmental assistance), teacher-related (e.g., related to teachers' beliefs, skills and confidence) or student-related (e.g., lack of student motivation and interest) [48]. In comparison to elementary schools, fewer teachers-related barriers are reported in secondary school studies where a lack of student motivation and interest emerge as barriers in PE [49]. Evidence suggests that motivation for physical activity engagement may change with maturation [50]. Intrinsic motivation (i.e., enjoyment of the activity) appears to play a leading role in physical activity participation among children, while other forms of autonomous motivations such as identified regulation (i.e., the outcome is identified as personally important), become more important among adolescents [50] and adults [51]. Numerous cross-sectional studies have identified positive associations between controlled and autonomous forms of motivation and physical activity in young people [52], yet empirical studies demonstrating the effect of school-based interventions on student motivation is lacking. There is a clear need for high quality experimental research to evaluate the impact of teacher professional learning interventions on secondary school students' motivation and MVPA in PE lessons [50] and also to determine any subsequent effects on leisure-time physical activity.

\section{Risk of bias}

The representativeness of the school, class and student sample were the primary sources of potential bias as limited information was provided to determine whether each of these samples were representative of the target population and the studies representative of usual PE lessons. This is not surprising given that many of the studies provided opportunistic data from trials rather than a purposeful sample for PE lesson proportion estimation. As previously noted, all middle school studies were conducted in the USA and the generalisability of findings to other countries for this age group is unclear.

Varied definitions of 'reporting time' between different instruments made it challenging to compare studies and may explain some of the differences in findings. Some studies calculated the proportion of MVPA time using the time of the total scheduled PE lesson (e.g., a 60-min lesson) while other studies only monitored physical activity for the time when students are engaged in activity or when a specified proportion of the class are in attendance. For example, accelerometer-assessed lessons typically monitor MVPA for the duration of the scheduled lesson based on school timetables, while the SOFIT observational method monitors student physical activity levels once $51 \%$ of students are present, and concludes when $51 \%$ of students have left the lesson [53]. Inconsistencies in reporting time, whether stated or not, may distort the results and provide an inaccurate representation of true MVPA time to compare against CDC recommendations [17]. Each instrument also measures different elements of activity to calculate MVPA. For example; accelerometers measure activity using the number of counts above certain cut-points, pedometers according to the number of steps/min, heart rate monitors according to heart rate levels above certain cut-points, and SOFIT uses movement categories. Some forms of activity may be categorised differently depending on the instrument used; for example, SOFIT classifies walking as MVPA while a non-brisk walking pace measured using accelerometers is unlikely to be considered MVPA [17]. Studies that used accelerometers to measure physical activity used different accelerometer cut-points to define MVPA (e.g., >1500 [37], >1962 [24] and >2001 [41] counts/min), which may have also contributed to the variation in findings between studies and made it difficult to compare and summarise the findings.

\section{Strengths and limitations}

This review provides a systematic synthesis of progress in achieving physically active PE lessons. The review included objectively-measured physical activity lessons and also included the addition of pedometerassessed physical activity in PE lessons. Two moderator analyses were conducted to assess if MVPA differed according to middle or high school PE lesson and type of measurement method. A meta-analysis was conducted to provide an overall pooled estimate of MVPA in secondary schools, as well as for the moderator analyses. The systematic review has some limitations. Seven studies did not provide adequate data to be pooled in to the meta-analysis and, despite several attempts, the study authors were unable to be contacted or were unable to provide the necessary additional information and data. In addition, a further three studies provided data based on one lesson and could not be included in the meta-analysis. As a result, only 15 of the 25 studies were included in the meta-analysis. We did not include any inclusion criteria about probability sampling for any of the study designs. The review was limited to studies that were published in English and in prominent databases. This may not be inclusive of all studies investigating MVPA in PE and published between 2005 and 2014. Studies related to MVPA in PE may appear in a broad range of journals including both educational and health fields, which may not always appear in prominent databases. We were unable to retrieve thesis and conference abstracts. 


\section{Recommendations for future research measuring activity levels in PE lessons}

Interventions designed to increase physically active learning time in PE lessons have been recommended as one potential mode of increasing overall MVPA in adolescents, and to promote lifelong activity [54, 55]. Intervention and observational studies investigating physical activity levels in PE lessons remain an important contribution to monitoring progress in the field. We offer the following recommendations to improve the quality of future research:

\section{Standardise the definition of 'PE lesson time':}

One solution could be reporting MVPA for lessons that monitor within a pre-specified proportion of the lesson (e.g., $\geq 90 \%$ ) separately from lessons that monitor a smaller proportion of the scheduled lesson (e.g., <90\%) [17]. Or at a minimum, state both the total allocated PE lesson duration and the monitoring time, if different.

2. Detailed reporting of MVPA outcomes: Comprehensive reporting is critical to fully monitor progress and maximise the number of studies that are eligible for inclusion in systematic reviews. At minimum, future observation and intervention studies should state the mean MVPA percentage of the lesson, a measure of variation (e.g., standard deviation), minutes of MVPA and the number of lessons examined so that data can be pooled into a meta-analysis.

3. Report types of physical activities: Future studies should include a clear description of the activities undertaken, and if possible, provide activity results separately for different types (e.g., fitness orientated, team invasion games, dance and gymnastics and net game activities). This may be challenging if different types of activity are undertaken within one lesson.

4. Ensure that PE lessons monitored are representative of usual PE lessons: To increase the representativeness of the findings, studies should monitor lessons from randomly selected schools and classes. All students within the lesson could be assessed, or a random sample of students monitored. Studies should aim to monitor numerous lessons (as many as feasible) as conclusions regarding the proportion of lesson time spent in MVPA can rarely be made from one observation. Information on the representativeness of the sample should also be provided.

5. Transparent and detailed reporting on study information: Many studies were excluded from the review as they lacked important information to confirm eligibility, and study authors were unable to be contacted. Reporting on the lesson context or structure (e.g., time spent in management, knowledge, skill practice, game play and fitness), content (e.g., type of PE activities), delivery (e.g., instructor behaviour) and environment (e.g., where the lesson is delivered and weather) will also enable more comprehensive analysis in future reviews.

Increasing activity levels in secondary school PE lessons $\mathrm{PE}$ is an opportunity to help students meet the $60 \mathrm{~min}$ of MVPA/day recommendation. Maximising MVPA in the existing scheduled PE lesson time could be achieved through targeted strategies such as i) teacher professional learning focused on reducing time spent in class management, instruction and organisation and optimising student motivation, and ii) by supplementing usual PE lessons with high intensity activity such as fitness infusion [54, 56-58]. Incorporating new technologies (e.g., Bluetooth pedometers that sync with apps) that provide real time individualised feedback and summaries of lesson physical activity levels could be tested as a strategy to motivate students and enable teachers to monitor MVPA during PE. Beets et al. [59] have proposed that future youth physical activity promotion interventions be designed according to a framework referred to as the Theory of Expanded, Extended and Enhanced Opportunities (TEO) using one or more of three mechanistic approaches: i) expansion - replacing time allocated for low active/sedentary activities with a new occasion to be active, ii) extension - increasing the time allocated for physical activity, and/or iii) enhancement - modifying existing physical activity opportunities to increase to amount of activity accumulated during an existing period of time. Whatever approach is taken, it is important that PE teachers are supported to balance the need of achieving active PE lessons while also meeting other curriculum and PE educational objectives. Meeting the 50\% MVPA target is only one aspect of measuring the quality of PE lessons. Although the MVPA target could be achieved by asking students to run continuous laps of an oval or gym, it is unlikely that this approach will engage students in meaningful learning experiences, and may negatively impact on student's attitudes, motivation and engagement in physical activity [17, 50, 54]. Not all PE lessons are conducive to high levels of MVPA but may still be, for example, valuable for skill development, fitness, knowledge of movements and improving social and emotional outcomes. For example, a gymnastics lesson may provide an opportunity for students to practice balance and rotation and involve cooperative learning, and may not meet the 50\% MVPA target.

\section{Conclusion}

Few studies specifically examining MVPA in PE lessons have been completed, and the studies that have been conducted are difficult to compare due to diverse MVPA measurement methods and incomplete reporting of MVPA outcomes. There is also limited data to comment 
on the generalisability of study findings. Based on the existing evidence, PE lessons in the secondary school setting fall short of the US CDC and UK AfPE recommendation of $50 \%$ of PE lesson time spent in MVPA. Although middle school PE lessons almost meet the MVPA recommendation, intervening in the middle school setting remains important in maintaining activity levels and ensuring that MVPA is undertaken equally across the student population. Further intervention research using the TEO framework [59] is needed to capitalise on building active lesson time in to existing PE lessons, working with schools to develop policies to ensure PE lesson time is protected (i.e., not cancelled) and/or extending the curriculum time allocated to PE. Future studies which monitor PE lessons in secondary schools should aim to incorporate the recommendations made in this review in to the study design to facilitate more accurate and comprehensive monitoring of MVPA in PE lessons.

\section{Additional file}

Additional file 1: A record of the search strategy used for each database. (DOCX 24 kb)

Additional file 2: 11-item risk of bias tool developed for the systematic review. (DOCX 14 kb)

\begin{abstract}
Abbreviations
95\% Cl: 95\% confidence intervals; AfPE: Associations for physical education; CDC: Centre for disease control; MVPA: Moderate to vigorous physical activity; non-RCTs: Non-randomised controlled trials; PE: Physical education; PRISMA: Preferred reporting items for systematic reviews and meta-analysis; RCTs: Randomised controlled trials; SD: Standard deviation; SE: Standard error; SES: Socioeconomic status; SOFIT: System for observing fitness instruction time; UK: United Kingdom; USA: United States of America
\end{abstract}

\section{Acknowledgements}

The authors would like to acknowledge Debbie Booth, a Research Librarian at the University of Newcastle, who assisted in refining the search strategy and conducted the database searches.

\section{Funding}

Infrastructure support was received from the Hunter Medical Research Institute (HMRI) and Hunter New England Population Health. DRL is supported by an Australian Research Council Future Fellowship.

\section{Availability of data and materials}

All data analysed during this study are included in this published article [and its Additional files].

\section{Authors' contributions}

All authors were responsible for the design of the study and the development of the search strategy. JLH and RS acted as first and second reviewer, respectively, and screened the studies, performed the risk of bias assessments and extracted study data. RS and JLH conducted the meta-analysis. EC acted as third reviewer, and resolved any disagreements during risk of bias assessments and data extraction. JLH, RS, DL, PM and JW developed the risk of bias tool. NN developed the data extraction tool. JLH drafted the introduction, methods and results section of the initial paper. JLH and RS drafted the discussion. All authors contributed to the interpretation of the results and all drafts of the manuscript. All authors read and approved the final manuscript.

\section{Competing interests}

The authors declare that they have no competing interests.

Consent for publication

Not applicable.

Ethical approval and consent to participate

Not applicable.

\section{Publisher's Note}

Springer Nature remains neutral with regard to jurisdictional claims in published maps and institutional affiliations.

\section{Author details}

${ }^{1}$ Hunter New England Population Health, Locked Bag 10, Wallsend, NSW 2287, Australia. ${ }^{2}$ School of Medicine and Public Health, University of Newcastle, Callaghan 2308, Australia. ${ }^{3}$ Hunter Medical Research Institute, Lambton, NSW 2305, Australia. ${ }^{4}$ Priority Research Centre in Physical Activity and Nutrition, School of Education, University of Newcastle, Callaghan 2308, Australia.

Received: 1 September 2016 Accepted: 5 April 2017

Published online: 24 April 2017

References

1. Gutin B, Yin Z, Humphries MC, Barbeau P. Relations of moderate and vigorous physical activity to fitness and fatness in adolescents. Am J Clin Nutr. 2005;81:746-50.

2. Ortega FB, Ruiz JR, Castillo MJ, Sjostrom M. Physical fitness in childhood and adolescence: a powerful marker of health. Int J Obes. 2007;32:1-11.

3. Sabiston CM, O'Loughlin E, Brunet J, Chaiton M, Low NC, Barnett T, et al. Linking depression symptom trajectories in adolescence to physical activity and team sports participation in young adults. Prev Med. 2013:56:95-8.

4. Biddle $\mathrm{SJH}$, Asare M. Physical activity and mental health in children and adolescents: a review of reviews. Br J Sports Med. 2011:45:886-95.

5. World Health Organisation. Global Strategy on Diet, Physical Activty and Health. In Physical Activity and Young People. 2014. http://www.who.int/dietphysicalactivity/ factsheet_young_people/en/index.html. Accessed 19th Nov 2015.

6. Hallal PC, Andersen LB, Bull FC, Guthold R, Haskell W, Ekelund U. Global physical activity levels: surveillance progress, pitfalls, and prospects. Lancet. 2012;380:247-7

7. Pate RR, Davis MG, Robinson TN, Stone EJ, McKenzie TL, Young JC. Promoting Physical activity in children and youth: a leadership role for schools: a scientific statement from the American Heart Association Council on nutrition, physical activity, and metabolism (physical activity committee) in collaboration with the councils on cardiovascular disease in the young and cardiovascular nursing. Circulation. 2006;114:1214-24.

8. Dumith SC, Gigante DP, Domingues MR, Kohl HW. Physical activity change during adolescence: a systematic review and a pooled analysis. Int J Epidemiol. 2011:40:685-98.

9. Centre for Disease Control and Prevention (CDC). Youth risk behaviour surveillance-United States 2009. In morbidity and mortality weekly report. 2010. www.cdc.gov/mmwr/preview/mmwrhtml/ss5905a1.htm. Accessed 5th Oct 2015.

10. U.S. Department of Health and Human Services, Centers for Disease Control and Prevention, National Center for Chronic Disease Prevention and Health Promotion, Division of Adolescent and School Health. Strategies to improve the quality of physical education. 2010. www.cdc.gov/healthyschools/pecat/ quality_pe.pdf. Accessed 23rd Oct 2015

11. Association for Physical Education (AfPE). Health Position Paper. 2015. http:// www.afpe.org.uk/physicaleducation/wp-content/uploads/afPE_Health_ Position_Paper_Web_Version2015.pdf. Accessed 11 Apr 2017.

12. Fairclough SJ, Stratton G. Physical activity levels in middle and high school physical education: a review. Pediatr Exerc Sci. 2005;17:217-36.

13. Moher D, Liberati A, Tetzlaff J, Altman DG. Preferred reporting items for systematic reviews and meta-analyses: the PRISMA statement. Ann Intern Med. 2009:151:264-9.

14. Higgins JPT, Green S (editors). Cochrane handbook for systematic reviews of interventions version 5.1.0 [updated March 2011]. The cochrane collaboration, 2011. Available from www.handbook.cochrane.org. 
15. Higgins JP, Altman DG, Gøtzsche PC, Jüni P, Moher D, Oxman AD, et al. The cochrane collaboration's tool for assessing risk of bias in randomised trials. BMJ. 2011;343:5928.

16. Van Sluijs EM, McMinn AM, Griffin SJ. Effectiveness of interventions to promote physical activity in children and adolescents: systematic review of controlled trials. BMJ. 2007:335:703-7.

17. Hollis JL, Williams AJ, Sutherland R, Campbell E, Nathan N, Wolfenden L, et al. A systematic review and meta-analysis of moderate-to-vigorous physical activity levels in elementary school physical education lessons. Prev Med. 2016;86:34-54.

18. Scruggs PW. Middle school physical education physical activity quantification: a pedometer steps/min guideline. Res Q Exerc Sport. 2007;78:284-92.

19. Fairclough S, Stratton G. Improving health-enhancing physical activity in girls' physical education. Health Educ Res. 2005;20:448-57.

20. Fairclough SJ, Stratton G. Effects of a physical education intervention to improve student activity levels. Phys Educ Sport Pedagog. 2006;11:29-44

21. Hannon JC, Ratliffe T, Holt B, Thorn J. Activity levels and female students' views of a high school physical education flag football unit: coeducational and single gender settings. ICHPER - SD J Res. 2005;41:16-21.

22. Wang GY, Pereira B, Mota J. Indoor physical education measured by heart rate monitor: a case study in Portugal. J Sports Med Phys Fitness. 2005:45:171-7.

23. Bronikowski M. How much physical activity a week to improve the healthrelated fitness of Polish schoolchildren?/W jakim stopniu aktywnosc fizyczna w ciagu tygodnia poprawia sprawnosc fizyczna polskich uczniow? Physical Education \& Sport/Wychowanie Fizyczne i Sport. 2005: 49:219-223.

24. Ferreira FS, Mota J, Duarte JA. Patterns of physical activity in Portuguese adolescents. Evaluation during physical education classes through accelerometry. Arch Exerc Health Dis. 2014;4:280-5.

25. Sanders T, Cliff DP, Lonsdale C. Measuring adolescent boys' physical activity: Bout length and the influence of accelerometer epoch length. PLoS One. 2014;9: e92040. doi:10.1371/journal.pone.0092040.

26. Conley MM, Gastin PB, Brown H, Shaw C. Heart rate biofeedback fails to enhance children's ability to identify time spent in moderate to vigorous physical activity. J Sci Med Sport. 2011;14:153-8.

27. Fu Y, Gao Z, Hannon J, Shultz B, Newton M, Sibthorp J. Influence of a health-related physical fitness model on students' physical activity, perceived competence, and enjoyment. Percept Mot Skills. 2013;117:956-70.

28. Lonsdale C, Rosenkranz RR, Sanders T, Peralta LR, Bennie A, Jackson B, et al. A cluster randomized controlled trial of strategies to increase adolescents' physical activity and motivation in physical education: results of the Motivating Active Learning in Physical Education (MALP) trial. Prev Med. 2013;57:696-702

29. McKenzie TL, Catellier DJ, Conway T, Lytle LA, Grieser M, Webber LA, et al. Girls' activity levels and lesson contexts in middle school PE: TAAG baseline. Med Sci Sports Exerc. 2006;38:1229-35.

30. Rosenkranz RR, Lubans DR, Peralta LR, Bennie A, Sanders T, Lonsdale C. A cluster-randomized controlled trial of strategies to increase adolescents' physical activity and motivation during physical education lessons: the Motivating Active Learning in Physical Education (MALP) trial. BMC Public Health. 2012;12:834.

31. Vidoni $C$, Azevedo L, Eberline A. Effects of a group contingency strategy on middle school physical education students' heart rates. Eur Phys Educ Rev. 2012;18:78-96.

32. Coe DP, Pivarnik JM, Womack CJ, Reeves MJ, Malina RM. Effect of physical education and activity levels on academic achievement in children. Med Sci Sports Exerc. 2006;38:1515-9.

33. How YM, Whipp P, Dimmock J, Jackson B. The effects of choice on autonomous motivation, perceived autonomy support, and physical activity levels in high school physical education. J Teach Phys Educ. 2013:32:131-48.

34. Liu W, Nichols RA, Zillifro TD. Comparison and comparability: fitness tracking between youths with different physical activity levels. Meas Phys Educ Exerc Sci. 2013;17:295-309.

35. Young DR, Phillips JA, Yu T, Haythornthwaite JA. Effects of a life skills intervention for increasing physical activity in adolescent girls. Arch Pediatr Adolesc Med. 2006;160:1255-61.

36. Barroso CS, Kelder $\mathrm{SH}$, Springer AE, Smith $\mathrm{CL}$, Ranjit N, Ledingham $\mathrm{C}$, et al. Senate Bill 42: implementation and impact on physical activity in middle schools. J Adolesc Health. 2009;45:S82-90.

37. Gao Z, Oh H, Sheng H. Middle school students' body mass index and physical activity levels in physical education. Res Q Exerc Sport. 2011;82:145-50.
38. Springer AE, Kelder SH, Byrd-Williams CE, Pasch KE, Ranjit N, Delk JE, et al. Promoting energy-balance behaviors among ethnically diverse adolescents: overview and baseline findings of The Central Texas CATCH middle school project. Health Educ Behav. 2013;40:559-70.

39. Dudley DA, Okely AD, Cotton WG, Pearson P, Caputi P. Physical activity levels and movement skill instruction in secondary school physical education. J Sci Med Sport. 2012;15:231-7.

40. Dudley DA, Okely AD, Pearson P, Cotton WG, Caputi P. Changes in physical activity levels, lesson context, and teacher interaction during physical education in culturally and linguistically diverse Australian schools. Int J Behav Nutr Phys Act. 2012;9:114.

41. Kremer MM, Reichert FF, Hallal PC. Intensity and duration of physical efforts in physical education classes. Rev Saude Publica. 2012;46:320-6.

42. Lonsdale C, Sabiston CM, Raedeke TD, Ha ASC, Sum RKW. Self-determined motivation and students' physical activity during structured physical education lessons and free choice periods. Prev Med. 2009;48:69-73.

43. Surapiboonchai K, Furney SR, Reardon RF, Eldridge J, Murray TD. SAM: a tool for Measurement of Moderate to Vigorous Physical Activity (MVPA) in school physical education. Int J Exerc Sci. 2012;5:127-35.

44. Owen $\mathrm{KB}$, Astell-Burt $\mathrm{T}$, Lonsdale $\mathrm{C}$. The relationship between selfdetermined motivation and physical activity in adolescent boys. J Adolesc Health. 2013:53:420-2.

45. Chow BC, McKenzie TL, Louie L. Physical activity and environmental influences during secondary school physical education. J Teach Phys Educ. 2009;28:21-37.

46. Scruggs PW, Mungen JD, Oh Y. Physical activity measurement device agreement: pedometer steps/minute and physical activity time. Meas Phys Educ Exerc Sci. 2010;14:151-63.

47. Scruggs PW, Mungen JD, Oh Y. Quantifying moderate to vigorous physical activity in high school physical education: a pedometer steps/minute standard. Meas Phys Educ Exerc Sci. 2010;14:104-15.

48. Morgan PJ, Hansen V. Classroom teachers' perceptions of the impact of barriers to teaching physical education on the quality of physical education programs. Res Q Exerc Sport. 2008;79:506-16.

49. Jenkinson KA, Benson AC. Barriers to providing physical education and physical activity in Victorian state secondary schools. Aust J Teach Edu (Online). 2010:35:1.

50. Owen KB, Smith J, Lubans DR, Ng JY, Lonsdale C. Self-determined motivation and physical activity in children and adolescents: a systematic review and meta-analysis. Prev Med. 2014;67:270-9.

51. Teixeira PJ, Carraça EV, Markland D, Silva MN, Ryan RM. Exercise, physical activity, and self-determination theory: a systematic review. Int J Behav Nutr Phys Act. 2012;9:1.

52. Braithwaite $R$, Spray CM, Warburton VE. Motivational climate interventions in physical education: a meta-analysis. Psychol Sport Exerc. 2011;12:628-38.

53. McKenzie TL, Sallis J, Nader P. System for observing fitness instruction time. J Teach Phys Educ. 1991;11:195-205.

54. Lonsdale C, Rosenkranz RR, Peralta LR, Bennie A, Fahey P, Lubans DR. A systematic review and meta-analysis of interventions designed to increase moderate-to-vigorous physical activity in school physical education lessons. Prev Med. 2013;56:152-61.

55. Sallis JF, McKenzie TL. Physical education's role in public health. Res Q Exerc Sport. 1991;62:124-37.

56. Smith NJ, Lounsbery MA, McKenzie TL. Physical activity in high school physical education: impact of lesson context and class gender composition. J Phys Act Health. 2014;11:127-35.

57. Senne T, Rowe D, Boswell B, Decker J, Douglas S. Factors associated with adolescent physical activity during middle school physical education: a one-year case study. Eur Phys Educ Rev. 2009:15:295-314.

58. Smith NJ, Monnat SM, Lounsbery MAF. Physical activity in physical education: are longer lessons better? J Sch Health. 2015:85:141-8.

59. Beets MW, Okely A, Weaver RG, Webster C, Lubans D, Brusseau T, et al. The theory of expanded, extended, and enhanced opportunities for youth physical activity promotion. Int J Behav Nutr Phys Act. 2016;13:120. 